\title{
Spectrum of a Feinberg-Zee random hopping matrix
}

\author{
Simon N. Chandler-Wilde and E. Brian Davies
}

\begin{abstract}
This paper provides a new proof of a theorem of Chandler-Wilde, Chonchaiya, and Lindner that the spectra of a certain class of infinite, random, tridiagonal matrices contain the unit disc almost surely. It also obtains an analogous result for a more general class of random matrices whose spectra contain a hole around the origin. The presence of the hole forces substantial changes to the analysis.
\end{abstract}

Mathematics Subject Classification (2010). 65F15, 15A18, 15A52, 47A10, 47A75, 47B80, $60 \mathrm{H} 25$.

Keywords. Spectrum, random matrix, hopping model, tridiagonal matrix, non-self-adjoint operator.

\section{Introduction}

Over the last fifteen years there have been many studies of the spectral properties of non-self-adjoint, random, tridiagonal matrices $A$, some of them cited in [8], [13], [14], and [17]. It has become clear that if all of the off-diagonal entries $A_{i, j}$ with $i-j= \pm 1$ of the matrices concerned are positive, the almost sure limit as $N \rightarrow \infty$ of the spectra of random $N \times N$ matrices subject to periodic boundary conditions can be quite different from the spectral behaviour of the corresponding infinite random matrix; see [9], [10], [15], and [16]. Indeed the limit in the first case can be the union of a small number of simple curves, while the second limit has a non-empty interior.

Numerical calculations suggest that the situation is quite different if the offdiagonal entries have variable signs, but much less has been proved in this situation, which is the one that we consider here. In a recent paper, [5], Chandler-Wilde, Chonchaiya, and Lindner made important progress in determining the almost sure spectrum of a remarkably interesting class of non-self-adjoint, random, tridiagonal matrices introduced by Feinberg and Zee in [13], and sometimes called random hopping matrices, because the diagonal entries all vanish. Specifically they proved that, 
contrary to earlier conjectures, the infinite, tridiagonal matrix

$$
A_{c}=\left(\begin{array}{cccccc}
\ddots & \ddots & & & & \\
\ddots & 0 & 1 & & & \\
& c_{n-1} & 0 & 1 & & \\
& & c_{n} & 0 & 1 & \\
& & & c_{n+1} & 0 & \ddots \\
& & & & \ddots & \ddots
\end{array}\right)
$$

has spectrum that contains the unit disc almost surely; see [5]. The paper assumed that the entries $c_{n}$ are independent and identically distributed with values in $\{ \pm 1\}$.

In the present paper we assume that the entries $c_{n}$ are independent and identically distributed with values in $\{ \pm \sigma\}$ for some fixed $\sigma \in(0,1]$. We assume that the probability $p$ that $c_{n}=\sigma$ satisfies $0<p<1$; the corresponding probability measure on $\Omega_{\sigma}=\{ \pm \sigma\}^{\mathbb{Z}}$ is denoted by $\mu$. The matrix $A_{c}$ is identified with the bounded operator acting in the natural manner on $\ell^{2}(\mathbb{Z})$.

In Lemma 26 we prove that

$$
\operatorname{Spec}\left(A_{c}\right) \subseteq\{\lambda: 1-\sigma \leq|\lambda| \leq 1+\sigma\}
$$

by a perturbation argument. We also prove that

$$
\operatorname{Spec}\left(A_{c}\right) \subseteq\left\{x+i y:|x|+|y| \leq \sqrt{2\left(1+\sigma^{2}\right)}\right\}
$$

by obtaining a bound on the numerical range of $A_{c}$. There are currently no general techniques for identifying the precise forms of holes in the spectra of non-self-adjoint operators, and we have not done so here, but numerical calculations are consistent with the hypothesis that it is the intersection, $H_{\sigma}$, of two elliptical regions as defined in (36); see the figures at the end of Section 7. Little is known about the part of the spectrum of $A_{c}$ outside the unit disc even in the case $\sigma=1$, but numerical studies suggest that the boundary of the spectrum has a self-similar fractal structure in that case; see [5] and [17].

The main result of [5], that the spectrum contains the unit disc almost surely, is for the case that $\sigma=1$, when there is no hole in the spectrum. It depends upon the identification of a particular sequence $c \in \Omega_{1}$ such that the equation $A_{c} f=\lambda f$ has a bounded solution $f$ for every $\lambda \in \mathbb{C}$ such that $|\lambda|<1$.

Our Theorem 7 rederives the main result of [5], in which $\sigma=1$, but depends on a certain operator identity introduced in the next section. Our main result, Theorem 27, that the spectrum of $A_{c}$ contains that part of the unit disc which is not in $H_{\sigma}$, applies to all $\sigma \in(0,1)$. We give a second proof of this result in Theorem 34 , by combining some results for $\sigma=1$ with bounds on the Lyapunov exponents of certain transfer matrices. Both proofs depend, additionally, on results on the spectra of operators on 
$\ell^{2}(\mathbb{Z})$ which have different periodic structures on the positive and negative half-axes. They also both depend on explicit spectral calculations which we are able to carry out for certain operators $A_{c}$ with $c$ having arbitrarily large period.

Our main results, as just stated, concern the spectrum of the (bi-)infinite matrix $A_{c}$. In a shorter final section we spell out implications for the spectra of the corresponding semi-infinite and finite matrices, illustrating these observations with computations of the finite matrix spectra. In particular we show, by applying recent results of Lindner and Roch [19], that, unlike $A_{c}$, the semi-infinite matrix has no hole in its spectrum for $\sigma \in(0,1)$, but contains the unit disc for all $\sigma \in(0,1]$.

Let $E_{\sigma}$ denote the set of all $c \in \Omega_{\sigma}$ that are pseudo-ergodic in the sense of [10]. Precisely, $c \in E_{\sigma}$ if for every finite sequence $b:\{1, \ldots, n\} \rightarrow\{ \pm \sigma\}$ there exists $m \in \mathbb{Z}$ such that $b_{r}=c_{m+r}$ for all $r \in\{1, \ldots, n\}$. Such sequences $c$ are easy to construct without any reference to probability theory. The following facts, proved in [10], and rederived in [18] and [7] as an instance of the application of limit operator arguments, will be crucial in this paper.

Proposition 1. If $b, c \in E_{\sigma}$, then $\operatorname{Spec}\left(A_{b}\right)=\operatorname{Spec}\left(A_{c}\right)$. Let $S_{\sigma}$ denote this set, which is the main object of study in the paper. If $c \in \Omega_{\sigma}$, then $c \in E_{\sigma}$ almost surely with respect to the measure $\mu$. Finally

$$
S_{\sigma}=\bigcup_{b \in \Omega_{\sigma}} \operatorname{Spec}\left(A_{b}\right)
$$

To describe a further result we establish, for $N \in \mathbb{N}$ and $\sigma \in(0,1]$ let $\pi_{N, \sigma}$ denote the union of $\operatorname{Spec}\left(A_{c}\right)$ over all $c \in \Omega_{\sigma}$ that are periodic with period $\leq N$. Let

$$
\pi_{\infty, \sigma}=\bigcup_{N \in \mathbb{N}} \pi_{N, \sigma} .
$$

One obvious implication of the above proposition is that

$$
\pi_{\infty, \sigma} \subset S_{\sigma} .
$$

As is well-known, the set $\pi_{N, \sigma}$ is the union of eigenvalues of $N \times N$ matrices. (Precisely, it is the union, over all sequences $c$ and all $|\alpha|=1$, of the eigenvalues of the matrix $A_{c, \alpha}^{(N, \text { per) }}$ defined in (51) below; see (40) and [11]. For another, equivalent characterisation see Lemma 20.) This simple observation is useful, in that it provides a method for computing what prove to be large subsets of $S_{\sigma}$, and will be one component in our arguments.

An interesting question is whether $\pi_{\infty, \sigma}$ is dense in $S_{\sigma}$. We do not answer this question one way or the other, but our method of proof of Theorem 7, showing that the unit disc is a subset of $S_{1}$, as a by-product, and with some additional argument, leads to a proof that $\pi_{\infty, 1}$ is dense in the unit disc (Theorem 15).

For the sake of simplicity we will, throughout the rest of the paper, omit the subscript $\sigma$ in our notations if $\sigma=1$. We use $\mathbb{N}$ and $\mathbb{Z}_{+}$, respectively, as our notations for the sets of positive and non-negative integers. 


\section{An abstract theorem}

In this section we present an abstract theorem that might be interesting in other contexts. It will be applied in Section 3.

Let $A$ be a bounded linear operator acting on the Hilbert space $H$ and let $H=$ $H_{e} \oplus H_{o}$ be an orthogonal decomposition of $H$.

Lemma 2. If $A\left(H_{e}\right) \subseteq H_{o}$ and $A\left(H_{o}\right) \subseteq H_{e}$, then $H_{e}$ and $H_{o}$ are invariant under the action of $A^{2}$. If $B$ is the restriction of $A^{2}$ to $H_{e}$ and $M$ is the restriction of $A^{2}$ to $H_{o}$ then

$$
\operatorname{Spec}\left(A^{2}\right) \backslash\{0\}=\operatorname{Spec}(B) \backslash\{0\}=\operatorname{Spec}(M) \backslash\{0\} .
$$

If $A$ is invertible then

$$
\operatorname{Spec}\left(A^{2}\right)=\operatorname{Spec}(B)=\operatorname{Spec}(M) .
$$

Proof. The decomposition $H=H_{e} \oplus H_{o}$ allows one to write the operator $A$ in the form

$$
A=\left(\begin{array}{cc}
0 & X \\
Y & 0
\end{array}\right)
$$

where $X: H_{o} \rightarrow H_{e}$ and $Y: H_{e} \rightarrow H_{o}$. Therefore

$$
A^{2}=\left(\begin{array}{cc}
X Y & 0 \\
0 & Y X
\end{array}\right)
$$

This implies that $B=X Y$ and $M=Y X$. The second identity in (3) follows by some simple algebra that holds for any pair of bounded operators $X$ and $Y$, and the first identity is a trivial consequence.

If $A$ is invertible then (5) implies that $B$ and $M$ are also invertible; therefore (4) is equivalent to (3).

Theorem 3. Let $H=\ell^{2}(\mathbb{Z})$, let $H_{e}$ be the closed subspace of sequences whose supports are contained in the set of even integers, and let $H_{o}$ be the closed subspace of sequences whose supports are contained in the set of odd integers. Let A be a bounded operator on $H$ whose matrix satisfies $A_{r, s}=0$ for all $r, s$ such that $|r-s| \neq 1$. Then $A\left(H_{e}\right) \subseteq H_{o}$ and $A\left(H_{o}\right) \subseteq H_{e}$. Moreover the identities

$$
\operatorname{Spec}\left(A^{2}\right)=\operatorname{Spec}(B)=\operatorname{Spec}(M)
$$

are valid in either of the following two cases.

Case 1. $\left|A_{r, s}\right|=1$ for all $r, s$ such that $|r-s|=1$.

Case 2. There exist constants $\beta$, $\gamma$ such that $0<\beta<\gamma<\infty$ and $\left|A_{r, s}\right| \leq \beta$ if $r-s=1$, while $\left|A_{r, s}\right| \geq \gamma$ if $r-s=-1$. 
Proof. Case 1. An elementary calculation establishes that there exists a sequence

$$
f: \mathbb{Z} \longrightarrow \mathbb{C}
$$

such that $A f=0,\left|f_{2 n}\right|=1$ for all $n$ and $f_{2 n+1}=0$ for all $n$, so that $A$ and $B$ are not invertible viewed as operators on $\ell^{\infty}(\mathbb{Z})$, and thus not invertible as operators on $\ell^{2}(\mathbb{Z})$; see e.g. [21], Theorem 2.5.2. So $0 \in \operatorname{Spec}(A)$ and $0 \in \operatorname{Spec}(B)$. Similarly there exists a sequence $f: \mathbb{Z} \rightarrow \mathbb{C}$ such that $A f=0,\left|f_{2 n+1}\right|=1$ for all $n$ and $f_{2 n}=0$ for all $n$. Hence $0 \in \operatorname{Spec}(M)$. The result follows by combining this with (3).

Case 2. The operator $A_{L}$ associated with the matrix

$$
\left(A_{L}\right)_{r, s}= \begin{cases}A_{r, s} & \text { if } r-s=-1, \\ 0 & \text { otherwise }\end{cases}
$$

is invertible and satisfies $\left\|A_{L}^{-1}\right\| \leq \gamma^{-1}$. The operator $A_{R}=A-A_{L}$ satisfies $\left\|A_{R}\right\| \leq \beta$. Therefore $A$ is invertible with

$$
\left\|A^{-1}\right\|=\left\|A_{L}^{-1}\left(I+A_{R} A_{L}^{-1}\right)^{-1}\right\| \leq \frac{\gamma^{-1}}{1-\beta / \gamma}=\frac{1}{\gamma-\beta} .
$$

The proof is completed by applying (4).

\section{The case $\sigma=1$}

The following lemma was noted in [5].

Lemma 4. If $c \in \Omega$ then $\operatorname{Spec}\left(A_{c}\right)$ is invariant with respect to both of the maps $\lambda \rightarrow \bar{\lambda}$ and $\lambda \rightarrow-\lambda$. If $\lambda \in S$ then $\bar{\lambda}$ and $i \lambda$ lie in $S$. Hence $S$ is invariant under the dihedral symmetry group $D_{2}$ generated by these two maps.

Proof. The invariance of $\operatorname{Spec}\left(A_{c}\right)$ under complex conjugation follows directly from the fact that $A_{c}$ has real entries. If $D$ is the diagonal matrix with entries $D_{r, r}=(-i)^{r}$ for all $r \in \mathbb{Z}$ then $D A_{c} D^{-1}=i A_{-c}$, so

$$
\operatorname{Spec}\left(A_{c}\right)=i \operatorname{Spec}\left(A_{-c}\right) \text {. }
$$

Iterating this identity yields $\operatorname{Spec}\left(A_{c}\right)=-\operatorname{Spec}\left(A_{c}\right)$. This proves the first part of the lemma. The second part follows once one observes that $c \in E$ if and only if $-c \in E$.

The formulae in (7) are related to those in [5], Proposition 2.1, in a way that we will make explicit in Section 4. However, nothing resembling the following lemma appears in [5]. 
Lemma 5. Given $b \in \Omega$, let $c=\Gamma_{+}(b) \in \Omega$ be the unique sequence satisfying

$$
c_{0}=1, \quad c_{2 n}+c_{2 n+1}=0, \quad c_{2 n} c_{2 n-1}=b_{n},
$$

for all $n \in \mathbb{Z}$. Then $A_{c}^{2}$ is unitarily equivalent to $A_{b} \oplus M_{b}$ acting in $\ell^{2}(\mathbb{Z}) \oplus \ell^{2}(\mathbb{Z})$, where

$$
\left(M_{b} f\right)_{n}=-f_{n-1}+\left(c_{2 n+1}+c_{2 n+2}\right) f_{n}+f_{n+1}
$$

for all $f \in \ell^{2}(\mathbb{Z})$. Moreover

$$
\operatorname{Spec}\left(A_{c}^{2}\right)=\operatorname{Spec}\left(A_{b}\right)=\operatorname{Spec}\left(M_{b}\right) .
$$

Proof. One may write $\left(A_{c} f\right)_{n}=c_{n} f_{n-1}+f_{n+1}$ for all $n \in \mathbb{Z}$, or equivalently $A_{c}=V_{c} R+L$ where $(L f)_{n}=f_{n+1},(R f)_{n}=f_{n-1}$ and $\left(V_{c} f\right)_{n}=c_{n} f_{n}$ for all $f \in \ell^{2}(\mathbb{Z})$.

Therefore

$$
\begin{aligned}
A_{c}^{2} & =V_{c} R V_{c} R+L V_{c} R+V_{c} R L+L^{2} \\
& =X_{c} R^{2}+Y_{c}+L^{2}
\end{aligned}
$$

where $X_{c}$ and $Y_{c}$ are the diagonal matrices with diagonal entries

$$
\begin{aligned}
X_{c, n, n} & =c_{n} c_{n-1}, \\
Y_{c, n, n} & =c_{n}+c_{n+1} .
\end{aligned}
$$

The operator $A_{c}^{2}$ has two invariant subspaces

$$
H_{e}=\left\{f \in \ell^{2}(\mathbb{Z}): f_{2 n+1}=0 \text { for all } n \in \mathbb{Z}\right\}
$$

and

$$
H_{o}=\ell^{2}(\mathbb{Z}) \ominus H_{e} .
$$

After an obvious relabeling of the subscripts, the restriction of $A_{c}^{2}$ to $H_{e}$ equals $A_{b}$ while the restriction of $A_{c}^{2}$ to $H_{o}$ is equal to $M_{b}$, as defined in (8). The final statement of the lemma is now an application of Theorem 3, Case 1.

We will exploit extensively the formula $\operatorname{Spec}\left(A_{c}^{2}\right)=\operatorname{Spec}\left(A_{b}\right)$ which appears in the above lemma. The equation $\operatorname{Spec}\left(A_{b}\right)=\operatorname{Spec}\left(M_{b}\right)$ will not play a role in our subsequent arguments, but makes an intriguing connection between spectra of rather different tridiagonal operators. Extending this connection slightly, for $b \in \Omega$ define $c=\Gamma_{+}(b)$ and $\tilde{M}_{b}$ by

$$
\left(\tilde{M}_{b} f\right)_{n}=f_{n-1}+i^{n}\left(c_{2 n+1}+c_{2 n+2}\right) f_{n}+f_{n+1},
$$

for all $f \in \ell^{2}(\mathbb{Z})$. Then, arguing as we do above to show (6), we see that

$$
\operatorname{Spec}\left(\tilde{M}_{b}\right)=i \operatorname{Spec}\left(M_{b}\right) \text {. }
$$


In particular, in the case $b \in E$ when, by Lemma $4, i \operatorname{Spec}\left(M_{b}\right)=i \operatorname{Spec}\left(A_{b}\right)=$ $\operatorname{Spec}\left(A_{b}\right)$, we see that

$$
S=\operatorname{Spec}\left(A_{b}\right)=\operatorname{Spec}\left(\tilde{M}_{b}\right)
$$

Thus, in studying $S$, we are studying both the almost sure spectrum of the infinite hopping-sign matrix $A_{b}$ with respect to the measure $\mu$, and the almost sure spectrum, with respect to the same measure, of $\tilde{M}_{b}$, a discrete Schrödinger operator with a particular, complex random potential.

In the next lemma we define the square root of any non-zero complex number to be the root whose argument lies in $(-\pi / 2, \pi / 2]$.

Lemma 6. If $b \in \Omega$ and $c=\Gamma_{+}(b)$ then $\lambda \in \operatorname{Spec}\left(A_{b}\right)$ if and only if $\pm \sqrt{\lambda}$ both lie in $\operatorname{Spec}\left(A_{c}\right)$. If $\lambda \in S$ then $\pm \sqrt{\lambda}$ both lie in $S$.

Proof. Lemma 4 and Lemma 5 imply that the following statements are equivalent. $\lambda \in \operatorname{Spec}\left(A_{b}\right) ; \lambda \in \operatorname{Spec}\left(A_{c}^{2}\right)=\left(\operatorname{Spec}\left(A_{c}\right)\right)^{2}$; either $\sqrt{\lambda}$ or $-\sqrt{\lambda}$ lies in $\operatorname{Spec}\left(A_{c}\right)$; $\pm \sqrt{\lambda}$ both lie in $\operatorname{Spec}\left(A_{c}\right)$.

If $\lambda \in S$ and $b \in E$ then $\lambda \in \operatorname{Spec}\left(A_{b}\right)$ by Proposition 1. Lemma 5 implies that

$$
\lambda \in \operatorname{Spec}\left(A_{c}^{2}\right)=\left(\operatorname{Spec}\left(A_{c}\right)\right)^{2} \subseteq S^{2} .
$$

Therefore either $\sqrt{\lambda}$ or $-\sqrt{\lambda}$ lie in $S$. The proof is completed by applying Lemma 4 .

Theorem 7. The set $S$ contains

$$
\bigcup_{n \in \mathbb{Z}_{+}, r \in\left\{0, \ldots, 2^{n+2}\right\}} \mathrm{e}^{\pi i r / 2^{n+1}}\left[0,2^{1 / 2^{n}}\right] .
$$

Hence $S$ contains the unit disc in $\mathbb{C}$.

Proof. For $n=0$ the theorem states that

$$
[0,2] \times\{1, i,-1,-i\} \subset S .
$$

This follows by combining Lemma 4 with direct calculations of $\operatorname{Spec}\left(A_{c}\right)$ when $c_{n}=1$ for all $n \in \mathbb{Z}$ (in which case $\operatorname{Spec}\left(A_{c}\right)=[-2,2]$ ) and when $c_{n}=-1$ for all $n \in \mathbb{Z}$ (in which case $\operatorname{Spec}\left(A_{c}\right)=i[-2,2]$ ). For larger $n$ the first statement of the theorem follows by applying Lemma 6 inductively. The second statement is now a consequence of the fact that the set (9) is dense in the unit disc.

\section{The maps $\Gamma_{ \pm}$}

A crucial role has been played in the proofs above by the nonlinear map $\Gamma_{+}$on $\Omega$ introduced in Lemma 5, and this map will be key to the arguments that we make 
throughout this paper. And in fact a sequence which is almost a fixed point of $\Gamma_{+}$(in a sense made precise below Lemma 8) is central to the proof of Theorem 7 in [5], though the proof is quite different and no mapping $\Gamma_{+}$appears in [5].

The relationship between the above proof of Theorem 7 and that in [5] is clarified to some extent by the following. Building on the definition of $\Gamma_{+}$made above, let us define maps $\Gamma_{ \pm}: \Omega \rightarrow \Omega$ by $\Gamma_{ \pm}(b)=c$ where

$$
c_{0}= \pm 1, \quad c_{2 n}+c_{2 n+1}=0, \quad c_{2 n} c_{2 n-1}=b_{n},
$$

for all $n \in \mathbb{Z}$. We also define the space inversion symmetry $b \rightarrow \hat{b}$ by $\hat{b}_{n}=b_{1-n}$ for all $n \in \mathbb{Z}$.

Lemma 8. If $\Gamma_{ \pm}(b)=c$ then $\Gamma_{\mp}(\hat{b})=\hat{c}$. In particular $\Gamma_{ \pm}(c)=c$ if and only if $\Gamma_{\mp}(\hat{c})=\hat{c}$. Each of the equations $\Gamma_{ \pm}(c)=c$ has exactly one solution.

Proof. Let $c=\Gamma_{+}(b)$ and $d=\Gamma_{-}(\hat{b})$. Then $d_{0}=-1, d_{2 n}+d_{2 n+1}=0$, and $d_{2 n} d_{2 n-1}=\hat{b}_{n}=b_{1-n}$ for all $n \in \mathbb{Z}$. Therefore $\hat{d}_{0}=d_{1}=1$. Also

$$
\hat{d}_{2 n+1}+\hat{d}_{2 n}=d_{1-(2 n+1)}+d_{1-2 n}=d_{-2 n}+d_{1-2 n}=0
$$

and

$$
\hat{d}_{2 n} \hat{d}_{2 n-1}=d_{1-2 n} d_{1-(2 n-1)}=d_{2(1-n)-1} d_{2(1-n)}=\hat{b}_{1-n}=b_{n}
$$

for all $n \in \mathbb{Z}$. Therefore $\hat{d}=\Gamma_{+}(b)=c$ and $d=\hat{c}$.

The proof that $c=\Gamma_{-}(b)$ implies $d=\Gamma_{+}(\hat{b})$ is similar. The other statements of the lemma follow immediately.

This paper and [5] use three different special sequences. The sequences $c_{ \pm}$are defined by $\Gamma_{ \pm}\left(c_{ \pm}\right)=c_{ \pm}$. It follows directly from their definitions that $c_{+, 0}=1$ and $c_{+, 1}=-1$, while $c_{-, 0}=-1$ and $c_{-, 1}=1$. However

$$
c_{+, n}=c_{-, n}=c_{+, 1-n}=c_{-, 1-n}
$$

for all $n \neq 0,1$. The paper [5] uses the sequence $c_{e}$ such that $c_{e, 0}=c_{e, 1}=1$, while $c_{e, n}=c_{ \pm, n}$ for all other $n$. Because of the space inversion symmetry the use of $c_{+}$ or $c_{-}$in any proof is really a matter of convenience.

We now turn to the solution of the equation $A_{c} u=\lambda u$ where $u: \mathbb{Z} \rightarrow \mathbb{C}$ is an arbitrary sequence. The eigenvalue equation is equivalent to the second order recurrence equation

$$
u_{n+1}+c_{n} u_{n-1}=\lambda u_{n} .
$$

Lemma 9. Suppose that $c \in \Omega$ and $\hat{c}_{n}=c_{1-n}$ for all $n \in \mathbb{Z}$; that $u_{n+1}+c_{n} u_{n-1}=$ $\lambda u_{n}$ for some $\lambda \in \mathbb{C}$ and all $n \in \mathbb{Z}$ and $u_{0}=0, u_{1}=1$; and that $\hat{u}_{n+1}+\hat{c}_{n} \hat{u}_{n-1}=$ $\lambda \hat{u}_{n}$ for all $n \in \mathbb{Z}$ and $\hat{u}_{0}=0, \hat{u}_{1}=1$. Then $\left|\hat{u}_{n}\right|=\left|u_{-n}\right|$ for all $n \in \mathbb{Z}$. In particular $u_{n}$ is bounded as $n \rightarrow \infty$ if and only if $\hat{u}_{n}$ is bounded as $n \rightarrow-\infty$. 
Proof. If one puts $v_{n}=u_{-n}$ then

$$
\hat{c}_{n+1} v_{n+1}+v_{n-1}=c_{-n} u_{-n-1}+u_{-n+1}=\lambda u_{-n}=\lambda v_{n}
$$

for all $n \in \mathbb{Z}$. Define $a: \mathbb{Z} \rightarrow\{ \pm 1\}$ by $a_{0}=1$ and $a_{n} / a_{n-1}=\hat{c}_{n}$ for all $n \in \mathbb{Z}$. If one now puts $w_{n}=a_{n} v_{n}$ for all $n \in \mathbb{Z}$ then (11) implies

$$
w_{n+1}+\hat{c}_{n} w_{n-1}=\lambda w_{n}
$$

for all $n \in \mathbb{Z}$. Since $w_{0}=v_{0}=u_{0}=0$ it follows that there exist $\gamma$ such that $w_{n}=\gamma \hat{c}_{n}$ for all $n \geq 1$. But $\left|c_{n}\right|=1$ for all $n$, so one obtains $|\gamma|=1$ by evaluating this identity for $n=1$. Therefore $\left|\hat{u}_{n}\right|=\left|w_{n}\right|=\left|v_{n}\right|=\left|u_{-n}\right|$ for all $n \geq 1$.

Corollary 10. Let $u_{+}, u_{-}, u_{e}: \mathbb{Z} \rightarrow \mathbb{C}$ be the solutions of $u_{n+1}+c_{n} u_{n-1}=\lambda u_{n}$ for all $n \in \mathbb{Z}$ subject to $u_{0}=0$ and $u_{1}=1$, if c is put equal to $c_{+}, c_{-}, c_{e}$ respectively. Then $u_{+, n}=u_{e, n}$ for all $n \in \mathbb{Z}$. Moreover $\left|u_{-, n}\right|=\left|u_{e, n}\right|$ for all $n \in \mathbb{Z}$.

Proof. The first statement is proved by an elementary computation. For the second we use $c_{e}=\hat{c_{e}}$ and $c_{-}=\hat{c_{+}}$. Lemma 9 now yields

$$
\left|u_{-, n}\right|=\left|u_{+,-n}\right|=\left|u_{e,-n}\right|=\left|u_{e, n}\right|
$$

for all $n \in \mathbb{Z}$.

The main step in the proof of Theorem 7 in [5] is contained in the following proposition (we quote here the parts of [5], Proposition 2.1, which we use immediately or later in Section 8).

Proposition 11. Let $u_{e}$ be defined as in Corollary 10 and define $p_{i, j} \in \mathbb{Z}$ for $i, j \in \mathbb{N}$ by the formula

$$
u_{e, i}=\sum_{j=1}^{i} p_{i, j} \lambda^{j-1}
$$

with $p_{i, j}=0$ if $j>i$. Let $Y$ denote the set of $(i, j) \in \mathbb{N}^{2}$ such that $p_{i, j} \neq 0$. Then $p_{i, j} \in\{0,1,-1\}$ for all $i, j$ and $(i, j) \in Y$ if and only if one of the following holds:

(1) $i=j=1$;

(2) $i$ and $j$ are both even and $(i / 2, j / 2) \in Y$;

(3) $i$ and $j$ are both odd and $((i+1) / 2,(j+1) / 2) \in Y$;

(4) $i$ and $j$ are both odd and $((i-1) / 2,(j+1) / 2) \in Y$.

The following result is an immediate corollary of this proposition and Corollary 10, which together imply that $\left|u_{e, i}\right| \leq(1-|\lambda|)^{-1}$ for $i \in \mathbb{Z}$ and $|\lambda|<1$. 
Theorem 12. [5] As in Corollary 10, let $u_{e}: \mathbb{Z} \rightarrow \mathbb{C}$ be the solution of $u_{n+1}+$ $c_{n} u_{n-1}=\lambda u_{n}$ for all $n \in \mathbb{Z}$ subject to $u_{0}=0$ and $u_{1}=1$, with $c=c_{e}$. Then $u_{e} \in \ell^{\infty}(\mathbb{Z})$ for $|\lambda|<1$, so that $\operatorname{Spec}\left(A_{c_{e}}\right)$ contains the unit disc.

Since Corollary 10 has shown that $u_{+}=u_{e}$, it is clear from Theorem 12 that $\operatorname{Spec}\left(A_{c_{+}}\right)$also contains the unit disc. In fact this is precisely its spectrum.

Theorem 13. If $c_{+}$is the unique solution of $\Gamma_{+}(c)=c$ then

$$
\operatorname{Spec}\left(A_{c_{+}}\right)=\{z:|z| \leq 1\} .
$$

Proof. It remains only to show that $\operatorname{Spec}\left(A_{c_{+}}\right) \subset\{z:|z| \leq 1\}$. If $\lambda \in \operatorname{Spec}\left(A_{c_{+}}\right)$ then repeated applications of the first part of Lemma 6 yield $\lambda^{2^{n}} \in \operatorname{Spec}\left(A_{c_{+}}\right)$for all $n \geq 1$. Since the spectrum is a bounded set, it follows that $|\lambda| \leq 1$.

We will (rather arbitrarily) focus on the mapping $\Gamma_{+}$rather than $\Gamma_{-}$in the remainder of the paper. The following lemma, which shows that the set of periodic sequences is invariant under the action of $\Gamma_{+}$, will play a key role.

Lemma 14. If $b \in \Omega$ is periodic with period $N$, i.e. $b_{n+N}=b_{n}, n \in \mathbb{Z}$, then $c=\Gamma_{+}(b)$ is $4 N$-periodic. Conversely, if $b \in \Omega, c=\Gamma_{+}(b)$, and $c$ is $2 N$-periodic for some $N \in \mathbb{N}$, then $b$ is $N$-periodic.

Proof. First note that, if $c=\Gamma_{+}(b)$ and one defines $\tilde{c} \in \Omega$ by $\tilde{c}_{n}=c_{2 n}, n \in \mathbb{Z}$, then

$$
c=\Gamma_{+}(b) \Longleftrightarrow\left(\tilde{c}_{0}=1, \tilde{c}_{n}=-b_{n} \tilde{c}_{n-1}, c_{2 n+1}=-\tilde{c}_{n}, n \in \mathbb{Z}\right) .
$$

Therefore

$$
\tilde{c}_{m+n}=\tilde{c}_{m}(-1)^{n} \prod_{j=1}^{n} b_{m+j}
$$

for all $m \in \mathbb{Z}$ and $n \in \mathbb{N}$. If $b$ is $N$-periodic, then

$$
\tilde{c}_{m+2 N}=\tilde{c}_{m} \prod_{j=1}^{2 N} b_{m+j}=\tilde{c}_{m} \prod_{j=1}^{N} b_{m+j}^{2}=\tilde{c}_{m},
$$

for all $m \in \mathbb{Z}$. Therefore $c$ is $4 N$-periodic.

Conversely, if $c=\Gamma_{+}(b)$, for some $b \in \Omega$, and $c$ is $2 N$-periodic for some $N \in \mathbb{N}$, then $\tilde{c}$ is $N$-periodic and, from (12), it follows that $b$ is $N$-periodic.

To illustrate the above lemma, define $c^{-}, c^{+} \in \Omega$ by $c_{n}^{-}=-1, c_{n}^{+}=1$, for $n \in \mathbb{Z}$, and define the sequences $c^{(m,+)}, c^{(m,-)} \in \Omega$, for $m=0,1, \ldots$, by

$$
c^{(0, \pm)}=c^{ \pm}, \quad c^{(m, \pm)}=\Gamma_{+}\left(c^{(m-1, \pm)}\right), \quad m \in \mathbb{N} .
$$


Then explicit calculations of the action of $\Gamma_{+}$yield that $c^{(1,+)}=\Gamma_{+}\left(c^{+}\right)$is 4-periodic (but not periodic with any smaller period), with $c_{-1}^{(1,+)}=c_{0}^{(1,+)}=1, c_{1}^{(1,+)}=$ $c_{2}^{(1,+)}=-1$. On the other hand, $c^{(1,-)}=\Gamma_{+}\left(c^{-}\right)$is 2-periodic (and so also 4periodic), with $c_{n}^{(1,-)}=(-1)^{n}$ for all $n \in \mathbb{Z}$.

Both these calculations, of course, are consistent with the lemma, which implies that $c^{(m, \pm)}$ is $N$-periodic with $N=4^{m}$, so that, using the notation (1) (dropping $\sigma$ given that $\sigma=1$ ),

$$
\operatorname{Spec}\left(A_{c^{(m, \pm)}}\right) \subset \pi_{4^{m}}, \quad m=0,1, \ldots
$$

Although we do not have an explicit formula for the sequences $c^{(m, \pm)}$, it is easy to compute $\operatorname{Spec}\left(A_{c^{(m, \pm)}}\right)$. By Lemma 6 , if $c=\Gamma_{+}(b)$, then

$$
\operatorname{Spec}\left(A_{c}\right)=\left\{ \pm \sqrt{\lambda}: \lambda \in \operatorname{Spec}\left(A_{b}\right)\right\} .
$$

The proof of Theorem 7 begins with the observation that $\operatorname{Spec}\left(A_{c^{+}}\right)=[-2,2]$ and $\operatorname{Spec}\left(A_{c^{-}}\right)=i[-2,2]$. Combining this observation with (16) we easily prove by induction that

$$
\operatorname{Spec}\left(A_{c^{(m,+)}}\right)=\left\{r \mathrm{e}^{\pi i j / 2^{m}}: 0 \leq r \leq 2^{1 / 2^{m}}, j \in\left\{0, \ldots, 2^{m+1}-1\right\}\right\}
$$

and

$$
\operatorname{Spec}\left(A_{c^{(m,-)}}\right)=\mathrm{e}^{\pi i / 2^{m+1}} \operatorname{Spec}\left(A_{c^{(m,+)}}\right) .
$$

Combining equations (15), (17), and (18), we see that we have shown that

$$
\left\{r \mathrm{e}^{\pi i j / 2^{m}}: 0 \leq r \leq 2^{1 / 2^{m+1}}, j \in\left\{0, \ldots, 2^{m+2}-1\right\}\right\} \subset \pi_{4^{m}}, \quad m=0,1, \ldots
$$

Thus we have shown the following modification of Theorem 7 which, of course, by (2), has Theorem 7 as a corollary.

Theorem 15. The set $\pi_{\infty}$ contains the set (9), and so is dense in the unit disc in $\mathbb{C}$.

We know $\operatorname{Spec}\left(A_{c^{(m, \pm)}}\right)$ explicitly, but do not have explicit formulae for the sequences $c^{(m, \pm)}$. However we can show that $c^{(m, \pm)}$ converges pointwise to the sequence $c_{+}$, the unique fixed point of $\Gamma_{+}$, as $m \rightarrow \infty$. This is the content of the next two lemmas. We omit a proof of the first of these lemmas which is an easy consequence, by simple induction arguments, of the definition of $\Gamma_{+}$.

Lemma 16. If $b \in \Omega$ and $c=\Gamma_{+}(b)$, then $c_{0}=c_{+, 0}$ and $c_{1}=c_{+, 1}$. If, for some $N \in \mathbb{N}, b_{m}=c_{+, m}$ for $m=1, \ldots, N$, then also $c_{m}=c_{+, m}$ for $m=2, \ldots, 2 N+1$. If, for some $N \in \mathbb{Z}_{+}, b_{-m}=c_{+,-m}$ for $m=0,1, \ldots, N$, then $b_{-m}=c_{+,-m}$ for $m=1,2, \ldots, 2 N+2$. 
Lemma 17. Let $b \in \Omega$, and define $c^{(n)} \in \Omega$ for $n \in \mathbb{N}$ by $c^{(1)}=\Gamma_{+}(b)$ and $c^{(n+1)}=\Gamma_{+}\left(c^{(n)}\right), n \in \mathbb{N}$. Then, for $n \in \mathbb{N}$,

$$
c_{m}^{(n)}=c_{+, m}, \quad m=2-2^{n}, 3-2^{n}, \ldots, 2^{n}-1,
$$

so that $c^{(n)} \rightarrow c_{+}$pointwise and $A_{c^{(n)}}$ converges strongly to $A_{c_{+}}$as $n \rightarrow \infty$. Further,

$$
\operatorname{Spec}\left(A_{c^{(n)}}\right) \subset\left\{\lambda:|\lambda| \leq 2^{1 / 2^{n}}\right\} .
$$

Proof. The first equation follows by induction from Lemma 16. The second equation follows by induction from (16) and the trivial bound that $\operatorname{Spec}\left(A_{b}\right) \subset\{\lambda:|\lambda| \leq 2\}$, which holds for all $b \in \Omega$.

\section{The mapping $\Gamma_{\sigma,+}$}

For the rest of the paper we consider operators $A_{c}$ for which the coefficients $c_{n}$ take values in $\{ \pm \sigma\}$, where $0<\sigma \leq 1$; that is, in the notation we have introduced in the introduction, we assume that $c \in \Omega_{\sigma}$, for some $\sigma \in(0,1]$.

The mapping $\Gamma_{+}$that we have introduced continues to play an important role. We extend the mapping so that it operates on $\Omega_{\sigma^{2}}$, defining, for $\sigma \in(0,1]$,

$$
\Gamma_{\sigma,+}: \Omega_{\sigma^{2}} \longrightarrow \Omega_{\sigma}
$$

by

$$
\Gamma_{\sigma,+}(c)=\sigma \Gamma_{+}\left(\sigma^{-2} c\right) .
$$

In other words, for $b \in \Omega_{\sigma^{2}}, c=\Gamma_{\sigma,+}(b)$ is the unique sequence in $\Omega_{\sigma}$ satisfying

$$
c_{0}=\sigma, \quad c_{2 n}+c_{2 n+1}=0, \quad c_{2 n} c_{2 n-1}=b_{n} .
$$

Main properties of the mapping $\Gamma_{\sigma,+}$ for our purposes are contained in the following extension of Lemma 6 . We will need to refer to a number of circular annuli, and use in this lemma and subsequently the notation

$$
\llbracket a, b \rrbracket=\{\lambda: a \leq|\lambda| \leq b\} .
$$

Lemma 18. If $b \in \Omega_{\sigma^{2}}$ and $c=\Gamma_{\sigma,+}(b) \in \Omega_{\sigma}$, then

$$
\left(\lambda \in \operatorname{Spec}\left(A_{b}\right) \Longleftrightarrow\left( \pm \sqrt{\lambda} \in \operatorname{Spec}\left(A_{c}\right)\right) .\right.
$$

Hence

$$
\left(\lambda \in S_{\sigma^{2}}\right) \Longrightarrow\left( \pm \sqrt{\lambda} \in S_{\sigma}\right)
$$

and

$$
\left(\llbracket a, b \rrbracket \subseteq S_{\sigma^{2}}\right) \Longrightarrow\left(\llbracket a^{1 / 2}, b^{1 / 2} \rrbracket \subseteq S_{\sigma}\right) .
$$


Proof. We modify the calculations in Section 3. Lemma 4 is valid as it stands. In Lemma 5 we assume that $b \in \Omega_{\sigma^{2}}$, and define $c \in \Omega_{\sigma}$ by $c=\Gamma_{\sigma,+}(b)$, and apply Case 2 of Theorem 3 in place of Case 1. This leads to the conclusion $\operatorname{Spec}\left(A_{c}^{2}\right)=$ $\operatorname{Spec}\left(A_{b}\right)$ as in Lemma 6. (23) and (24) follow by choosing $b \in E_{\sigma^{2}}$ and using Proposition 1.

\section{Periodic and paired periodic operators}

To prove our main theorem we need results on operators $A_{c}$ on $\ell^{2}(\mathbb{Z})$ that have one periodic structure for $n \geq 0$ and another for $n<0$ (which we term paired periodic operators). The essential spectrum of such an operator is the union of the essential spectra of the periodic operators involved, which may be calculated explicitly using their Bloch decompositions.

There may also be substantial inessential spectrum, in particular, open subsets of the spectrum where $A_{c}-\lambda I$ is Fredholm but has non-zero index. These parts of the spectrum (and the corresponding values of the index) can be computed by application of general results for block Toeplitz operators, which have been developed to a high degree of sophistication; see [1], [3], [2], [4], and the references therein. We need only a small part of this theory, and it is easy to develop this from first principles. We do this in a short Lemma 25 below, inspired by earlier analysis in [12], [9], [10], and particularly Theorem 12 in [10]. Both the proof of Lemma 25, and the effective application of this lemma to prove Theorem 27, depend on the next two lemmas which describe properties of the spectra and eigenfunctions of periodic operators.

We assume throughout this section that the parameter $\sigma \in(0,1)$.

Lemma 19. Let

$$
\Phi(\tau, \gamma)=\frac{\operatorname{Re}(\tau)^{2}}{(1+\gamma)^{2}}+\frac{\operatorname{Im}(\tau)^{2}}{(1-\gamma)^{2}},
$$

where $\tau \in \mathbb{C}$ and $-1<\gamma<1$. Then the quadratic equation

$$
z^{2}-\tau z+\gamma=0
$$

has a solution satisfying $|z|=1$ if and only if $\Phi=1$. If $\Phi<1$ then both solutions satisfy $|z|<1$. If $\Phi>1$ then one solution satisfies $|z|<1$ and the other satisfies $|z|>1$.

Proof. For $\theta \in \mathbf{R}, z=\mathrm{e}^{i \theta}$ is a solution of (26) if and only if

$$
\cos (\theta)=\frac{\operatorname{Re}(\tau)}{1+\gamma}, \quad \sin (\theta)=\frac{\operatorname{Im}(\tau)}{1-\gamma},
$$

so that (26) has a solution satisfying $|z|=1$ if and only if $\Phi(\tau, \gamma)=1$. 
The set $U=\{\tau \in \mathbb{C}: \Phi<1\}$ is connected and contains the origin. Since the solutions of (26) depend continuously on $\tau$, and both solutions satisfy $|z|<1$ if $\tau=0$, it follows that both satisfy $|z|<1$ for all $\tau \in U$. The case $\Phi>1$ is similar.

The following lemma is closely related to a similar result for the non-self-adjoint Anderson model in [10], Theorem 11.

Lemma 20. If $c \in \Omega_{\sigma}$ and $\lambda \in \mathbb{C}$ then the space of all solutions of $A_{c} f=\lambda f$ is two-dimensional. If $c$ is periodic with period $p$ then the asymptotic behaviour as $n \rightarrow \pm \infty$ of the solutions is determined by the solutions $z_{1}, z_{2}$ of the polynomial $z^{2}-\tau(\lambda) z+\gamma=0$, where $\tau(\lambda)$ is a monic polynomial in $\lambda$ with degree $p$, given by $\tau(\lambda)=\operatorname{tr}\left(T_{p}\right)$, where $T_{p}=X_{p} X_{p-1} \ldots X_{1}$ and

$$
X_{n}=\left(\begin{array}{cc}
0 & 1 \\
-c_{n} & \lambda
\end{array}\right),
$$

and $\gamma=\operatorname{det}\left(T_{p}\right)= \pm \sigma^{p}$. Ordering the two solutions so that $\left|z_{1}\right| \geq\left|z_{2}\right|$, there are three cases.

(1) $\lambda$ lies in the closed set

$$
B_{c}=\left\{\lambda:\left|z_{1}\right|=1 \text { and }\left|z_{2}\right|=\sigma^{p}\right\} .
$$

This set is the spectrum of $A_{c}$, equivalently, the set of $\lambda$ for which $A_{c} f=\lambda f$ has a bounded solution.

(2) $\lambda$ lies in the open set

$$
I_{c}=\left\{\lambda: 1>\left|z_{1}\right| \geq\left|z_{2}\right|>\sigma^{p}\right\} .
$$

This is the case if and only if all solutions of $A_{c} f=\lambda f$ decay exponentially as $n \rightarrow+\infty$.

(3) $\lambda$ lies in the open set

$$
O_{c}=\left\{\lambda:\left|z_{1}\right|>1 \text { and }\left|z_{2}\right|<\sigma^{p}\right\} .
$$

This is the case if and only if there exists a solution of $A_{c} f=\lambda f$ that decays exponentially as $n \rightarrow+\infty$ and grows exponentially as $n \rightarrow-\infty$, and another solution that decays exponentially as $n \rightarrow-\infty$ and grows exponentially as $n \rightarrow+\infty$.

Proof. The sequence $f: \mathbb{Z} \rightarrow \mathbb{C}$ is a solution of $A_{c} f=\lambda f$ if and only if $f_{n+1}+$ $c_{n} f_{n-1}=\lambda f_{n}$ for all $n \in \mathbb{Z}$. This recurrence relation can be rewritten in the form

$$
\left(\begin{array}{c}
f_{n} \\
f_{n+1}
\end{array}\right)=\left(\begin{array}{cc}
0 & 1 \\
-c_{n} & \lambda
\end{array}\right)\left(\begin{array}{c}
f_{n-1} \\
f_{n}
\end{array}\right)
$$




$$
\begin{aligned}
& =X_{n}\left(\begin{array}{c}
f_{n-1} \\
f_{n}
\end{array}\right) \\
& =T_{n}\left(\begin{array}{c}
f_{0} \\
f_{1}
\end{array}\right),
\end{aligned}
$$

where $T_{n}=X_{n} X_{n-1} \ldots X_{1}$. If $c$ is periodic with period $p$, then the asymptotic behaviour of the two-dimensional space of eigenfunctions $f$ is determined by the magnitude of the eigenvalues $z_{1}, z_{2}$ of $T_{p}$. These are the solutions of the equation $z^{2}-\tau z+\gamma=0$ where $\tau=\operatorname{tr}\left(T_{p}\right)$ and $\gamma=\operatorname{det}\left(T_{p}\right)$. A simple induction establishes that the $(i, j)$-th entry of $T_{p}$ is a polynomial in $\lambda$ with degree less than $p$ unless $i=j=2$ in which case it is a monic polynomial with degree $p$. Therefore $\tau$ is a monic polynomial in $\lambda$ with degree $p$. However

$$
\operatorname{det}\left(T_{p}\right)=\prod_{r=1}^{p} \operatorname{det}\left(X_{r}\right)=c_{1} \ldots c_{p}= \pm \sigma^{p}
$$

does not depend on $\lambda$. The continuous dependence of the roots of a polynomial on its coefficients implies that $B_{c}$ is closed while $I_{c}$ and $O_{c}$ are open. An application of Lemma 19 now completes the proof. One sees, in particular, that

$$
\operatorname{Spec}\left(A_{c}\right)=B_{c}=\{\lambda: \Phi(\tau, \gamma)=1\} .
$$

Our next lemma enables us to determine the sets $I_{c}$ and $O_{c}$ for certain important periodic sequences $c$, and to determine the spectra of certain paired periodic operators. We continue with the assumptions and notation of Lemma 20.

Lemma 21. If $V$ is a connected component of $\mathbb{C} \backslash B_{c}$ then $V \subseteq I_{c}$ or $V \subseteq O_{c}$. If $V$ is unbounded then $V \subseteq O_{c}$, and if $0 \in V$ then $V \subseteq I_{c}$. If $\mathbb{C} \backslash B_{c}$ has exactly two components then the bounded component equals $I_{c}$ and the unbounded component equals $O_{c}$.

Proof. We first observe that $V, I_{c}$, and $O_{c}$ are all open sets and that their definitions imply directly that $I_{c}$ and $O_{c}$ are disjoint. Therefore $V=\left(V \cap I_{c}\right) \cup\left(V \cap O_{c}\right)$, where the two intersections on the right-hand side are disjoint. Since $V$ is connected, it follows that $V=V \cap I_{c}$ or $V=V \cap O_{c}$ This completes the proof of the first statement.

Lemma 20(1) implies that

$$
B_{c}=\operatorname{Spec}\left(A_{c}\right) \subseteq\{\lambda:|\lambda| \leq 1+\sigma\} .
$$

Therefore $\mathbb{C} \backslash B_{c}$ has only one unbounded component $V$ and it contains $\{\lambda:|\lambda|>$ $1+\sigma\}$. To prove that $V \subseteq O_{c}$ it is sufficient by the first part of this proof to find a single point $\lambda \in V \cap O_{c}$. The fact that $\tau$ is a polynomial with degree $p$ implies that $|\tau(\lambda)| \rightarrow \infty$ as $|\lambda| \rightarrow \infty$. This implies that the solutions of $z^{2}-\tau(\lambda) z+\gamma=0$, 
where $\gamma= \pm \sigma^{p}$, are $z \sim \tau(\lambda)$ and $z \sim \gamma / \tau(\lambda)$ to leading order for all large enough $|\lambda|$. Therefore $\lambda \in O_{c}$ for all such $\lambda$.

The proof is completed by proving that $0 \in I_{c}$. For $\lambda=0$ one has $T_{p}=$ $X_{p} X_{p-1} \ldots X_{1}$ where each $X_{r}$ is of the form $\left(\begin{array}{cc}0 & 1 \\ \pm \sigma & 0\end{array}\right)$. If $p=2 m$ it follows that $T_{p}=\left(\begin{array}{cc} \pm \sigma^{m} & 0 \\ 0 & \pm \sigma^{m}\end{array}\right)$. The fundamental equation must therefore take one of the forms $z^{2}-2 \sigma^{m} z+\sigma^{2 m}=0, z^{2}+2 \sigma^{m} z+\sigma^{2 m}=0$, or $z^{2}-\sigma^{2 m}=0$. In each case both solutions have modulus $\sigma^{p / 2}<1$. The same holds if $p=2 m+1$.

The final statement of the lemma is a consequence of the following observations. There must be a component of $\mathbb{C} \backslash B_{c}$ that contains 0 and there must be an unbounded component. The first part of the proof shows that these are distinct, and the extra hypothesis is that there are no other components.

Our next task is to determine the sets $B_{c}, I_{c}$, and $O_{c}$ for certain particular periodic sequences.

Lemma 22. If $c_{n}=\sigma$ for all $n \in \mathbb{Z}$ then $\operatorname{Spec}\left(A_{c}\right)$ is the ellipse

$$
\begin{aligned}
\operatorname{Spec}\left(A_{c}\right) & =\left\{u+i v: \frac{u^{2}}{(1+\sigma)^{2}}+\frac{v^{2}}{(1-\sigma)^{2}}=1\right\} \\
& =\left\{\rho \mathrm{e}^{i \theta}: \rho=\frac{1-\sigma^{2}}{\sqrt{1+\sigma^{2}-2 \sigma \cos (2 \theta)}}\right\} .
\end{aligned}
$$

Moreover the interior $U$ of the ellipse equals $I_{c}$ and the exterior $V$ of the ellipse equals $O_{c}$.

Proof. We have $p=1$ and $T_{1}=\left(\begin{array}{cc}0 & 1 \\ -\sigma & \lambda\end{array}\right)$, so $\tau(\lambda)=\lambda$ and $\gamma=\sigma$. Using (25) we deduce that $\operatorname{Spec}\left(A_{c}\right)$ is given by (27). The proof is completed by using Lemma 21 .

Lemma 23. If $c_{n}=-\sigma$ for all $n \in \mathbb{Z}$ then $\operatorname{Spec}\left(A_{c}\right)$ is the ellipse

$$
\begin{aligned}
\operatorname{Spec}\left(A_{c}\right) & =\left\{u+i v: \frac{u^{2}}{(1-\sigma)^{2}}+\frac{v^{2}}{(1+\sigma)^{2}}=1\right\} \\
& =\left\{\rho \mathrm{e}^{i \theta}: \rho=\frac{1-\sigma^{2}}{\sqrt{1+\sigma^{2}+2 \sigma \cos (2 \theta)}}\right\} .
\end{aligned}
$$

Moreover $I_{c}$ is the interior of the ellipse and $O_{c}$ is the exterior of the ellipse.

Proof. We have $p=1$ and $T_{1}=\left(\begin{array}{cc}0 & 1 \\ \sigma & \lambda\end{array}\right)$, so $\tau(\lambda)=\lambda$ and $\gamma=-\sigma$. We omit the rest of proof, which is almost identical to that of Lemma 22.

In the following lemma, starting from Lemmata 22 and 23, and making successive applications of Lemma 18, we compute the spectra of a family of periodic sequences, 
namely the sequences $c^{ \pm}=\sigma c^{(n, \pm)} \in \Omega_{\sigma}$, defined by (14). By Lemma 14 , these sequences are periodic of period $\leq 4^{n}$.

This next lemma applies for $0<\sigma<1$. The corresponding result for $\sigma=1$ is equations (17) and (18) above.

Lemma 24. Suppose $n \in \mathbb{Z}_{+}$and $c^{+}=\sigma c^{(n,+)}, c^{-}=\sigma c^{(n,-)}$. Then

$$
\operatorname{Spec}\left(A_{c^{ \pm}}\right)=\left\{\rho \mathrm{e}^{\mathrm{i} \theta}: \rho=\rho_{n}^{ \pm}(\theta, \sigma)\right\}
$$

where

$$
\begin{aligned}
& \rho_{0}^{+}(\theta, \sigma)=\frac{1-\sigma^{2}}{\left(1+\sigma^{2}-2 \sigma \cos 2 \theta\right)^{1 / 2}}, \\
& \rho_{0}^{-}(\theta, \sigma)=\frac{1-\sigma^{2}}{\left(1+\sigma^{2}+2 \sigma \cos 2 \theta\right)^{1 / 2}},
\end{aligned}
$$

and, for $n \in \mathbb{N}$,

$$
\rho_{n}^{ \pm}(\theta, \sigma)=\left(\rho_{0}^{ \pm}\left(2^{n} \theta, \sigma^{2^{n}}\right)\right)^{1 / 2^{n}}=\frac{\left(1-\sigma^{2^{n+1}}\right)^{1 / 2^{n}}}{\left(1+\sigma^{2^{n+1}} \mp 2 \sigma^{2^{n}} \cos \left(2^{n+1} \theta\right)\right)^{1 / 2^{n+1}} .}
$$

Moreover,

$$
I_{c^{ \pm}}=\left\{\rho \mathrm{e}^{i \theta}: 0 \leq \rho<\rho_{n}^{ \pm}(\theta, \sigma)\right\}
$$

and

$$
O_{c^{ \pm}}=\left\{\rho \mathrm{e}^{i \theta}: \rho>\rho_{n}^{ \pm}(\theta, \sigma)\right\} .
$$

Proof. Our proof of (31) is by induction. We note first that (31) holds for $n=0$ by Lemmata 22 and 23. Suppose now that (31) holds for some $n \geq 0$ and all $0<\sigma<1$. Then

$$
\begin{aligned}
\operatorname{Spec}\left(A_{\sigma^{2} c^{(n, \pm)}}\right) & =\left\{\rho \mathrm{e}^{\mathrm{i} \theta}: \rho=\rho_{n}^{ \pm}\left(\theta, \sigma^{2}\right)\right\} \\
& =\left\{\rho \mathrm{e}^{\mathrm{i} \theta}: \rho=\left(\rho_{0}^{ \pm}\left(2^{n} \theta, \sigma^{2^{n+1}}\right)\right)^{1 / 2^{n}}\right\} .
\end{aligned}
$$

Since $\sigma c^{(n+1, \pm)}=\sigma \Gamma_{+}\left(c^{(n,+)}\right)=\Gamma_{\sigma,+}\left(\sigma^{2} c^{(n,+)}\right)$, it follows from Lemma 18 that

$$
\operatorname{Spec}\left(A_{\sigma c^{(n+1, \pm)}}\right)=\left\{ \pm \sqrt{\lambda}: \lambda \in \operatorname{Spec}\left(A_{\sigma^{2} c^{(n, \pm)}}\right)\right\} .
$$

Combining these equations, we see that (31) holds with $n$ replaced by $n+1$. Thus (31) follows by induction.

The formulae for $I_{c^{ \pm}}$and $O_{c^{ \pm}}$follow from (31) and Lemma 21. 
We remark that $\rho_{n}^{-}(\theta, \sigma)=\rho_{n}^{+}\left(\theta \pm \pi / 2^{n+1}, \sigma\right)$, so that the spectra of $A_{c^{ \pm}}$in the above lemma are related by

$$
\operatorname{Spec}\left(A_{c^{+}}\right)=\mathrm{e}^{ \pm i \pi / 2^{n+1}} \operatorname{Spec}\left(A_{c^{-}}\right) .
$$

This is a symmetry which is surprising from an inspection of the sequences $c^{ \pm}$, which need not even have the same period. (For example, as observed in Section $4, c^{+}$has period 4 and $c^{-}$period 2 in the case $n=1$.)

In principle, since $c^{ \pm}$is periodic, (31) should be computable alternatively from the characterisation of the spectrum for general periodic sequences in Lemma 20. As an example of this, for the sequence $c^{-}=\sigma c^{(1,-)}$ which has period 2, with $c_{n}^{-}=(-1)^{n} \sigma$, the transfer matrix $T_{2}$ is given by

$$
T_{2}=X_{2} X_{1}=\left(\begin{array}{cc}
0 & 1 \\
-\sigma & \lambda
\end{array}\right)\left(\begin{array}{ll}
0 & 1 \\
\sigma & \lambda
\end{array}\right)=\left(\begin{array}{cc}
\sigma & \lambda \\
\sigma \lambda & -\sigma+\lambda^{2}
\end{array}\right) .
$$

Applying Lemmata 19 and 20 with $\tau=\lambda^{2}$ and $\gamma=-\sigma^{2}$, we find that $\operatorname{Spec}\left(A_{c}\right)$ is the set of all $\lambda=u+i v$ for which

$$
\frac{\left(u^{2}-v^{2}\right)^{2}}{\left(1-\sigma^{2}\right)^{2}}+\frac{(2 u v)^{2}}{\left(1+\sigma^{2}\right)^{2}}=1 \text {. }
$$

If one puts $\lambda=\rho \mathrm{e}^{i \theta}$, then this may be rewritten in the form (31).

The main point of the above theory and calculations are to prove and prepare the use of the following result on operators $A_{c}$ that are paired periodic operators. To state this result let us introduce the notations

$$
E_{\sigma}=\left\{x+i y: \frac{x^{2}}{(1+\sigma)^{2}}+\frac{y^{2}}{(1-\sigma)^{2}}<1\right\}
$$

and

$$
E_{-\sigma}=\left\{x+i y: \frac{x^{2}}{(1-\sigma)^{2}}+\frac{y^{2}}{(1+\sigma)^{2}}<1\right\},
$$

so that $E_{\sigma}$ and $E_{-\sigma}$ are the interiors of the ellipses introduced in Lemmata 22 and 23.

The following lemma is analogous to [10], Theorem 12, proved there for the non-self-adjoint Anderson model.

Lemma 25. Suppose that $c \in \Omega_{\sigma}$ is periodic and $\tau \in\{\sigma,-\sigma\}$, and define $c^{*} \in \Omega_{\sigma}$ by $c_{n}^{*}=c_{n}$, for $n \geq 0$, and $c_{n}^{*}=\tau$ for $n<0$. Then

$$
\operatorname{Spec}\left(A_{c^{*}}\right) \supset \overline{I_{c}} \backslash E_{\tau} .
$$

Proof. Since $\operatorname{Spec}\left(A_{c^{*}}\right)$ is closed it is enough to show that $\operatorname{Spec}\left(A_{c *}\right) \supset I_{c} \backslash \overline{E_{\tau}}$. So suppose that $\lambda \in I_{c} \backslash \overline{E_{\tau}}$. Then, by Lemmata 22 and 23 and Lemma 20, since 
$\lambda \notin \overline{E_{\tau}}$, it follows that there exists a non-trivial solution $f$ of $A_{c^{*}} f=\lambda f$ such that $f_{n} \rightarrow 0$ exponentially as $n \rightarrow-\infty$. Since $\lambda \in I_{c}$, again applying Lemma 20, it follows that this solution $f$ also decays exponentially as $n \rightarrow+\infty$. Thus $\lambda$ is an eigenvalue of $A_{c^{*}}$ so $\lambda \in \operatorname{Spec}\left(A_{c^{*}}\right)$.

\section{First proof of the main theorem}

This section is devoted to the proof of Theorem 27, in which we assume that $0<$ $\sigma<1$.

Lemma 26. We have

$$
\operatorname{Spec}\left(A_{c}\right) \subseteq \llbracket 1-\sigma, 1+\sigma \rrbracket
$$

and

$$
\operatorname{Spec}\left(A_{c}\right) \subseteq\left\{x+i y:|x|+|y| \leq \sqrt{2\left(1+\sigma^{2}\right)}\right\},
$$

for every choice of $c \in \Omega_{\sigma}$.

Proof. We regard $V_{c} R$ as a small perturbation of $L$ in the identity $A_{c}=V_{c} R+$ $L$, noted in the proof of Lemma 5 . Since $L$ is a unitary operator with spectrum $\{z:|z|=1\}$, we have

$$
\left\|(L-z I)^{-1}\right\|=|1-| z||^{-1}
$$

for all $z$ not on the unit circle. The inclusion (34) now follows from $\left\|V_{c} R\right\|=\sigma$ by a perturbation argument; see [11], Theorem 9.2.13.

The inclusion (35) depends on an estimate of the numerical range of $A_{c}$. Following [11], Section 9.3, $x+i y \in \operatorname{Num}\left(A_{c}\right)$ if there exists $f \in \ell^{2}(\mathbb{Z})$ such that $\|f\|=1$ and $x+i y=\left\langle A_{c} f, f\right\rangle$. This implies that

$$
\begin{aligned}
& x=\frac{1}{2}\left\langle\left(A_{c}+A_{c}^{*}\right) f, f\right\rangle, \\
& y=-\frac{i}{2}\left\langle\left(A_{c}-A_{c}^{*}\right) f, f\right\rangle .
\end{aligned}
$$

Therefore

$$
x+y=\frac{1}{2}\langle B f, f\rangle,
$$

where

$$
B=\left(A_{c}+A_{c}^{*}\right)-i\left(A_{c}-A_{c}^{*}\right) .
$$

A simple calculation shows that $B_{m, n}=0$ unless $|m-n|=1$, while

$$
B_{n, n+1}=\overline{B_{n+1, n}}=(1 \pm \sigma)-i(1 \mp \sigma) .
$$


Therefore $\left|B_{n+1, n}\right|=\left|B_{n, n+1}\right|=\sqrt{2\left(1+\sigma^{2}\right)}$ for all $n \in \mathbb{Z}$ and

$$
x+y \leq \frac{1}{2}\|B\| \leq \sqrt{2\left(1+\sigma^{2}\right)} .
$$

The other three steps in the proof of the bound for $|x|+|y|$ are similar.

The statement of our main theorem refers to the open set

$$
H_{\sigma}=E_{\sigma} \cap E_{-\sigma}
$$

the intersection of the ellipses $E_{\sigma}$ and $E_{-\sigma}$. This set satisfies

$$
\llbracket 0,1-\sigma \rrbracket \subseteq \overline{H_{\sigma}} \subseteq \llbracket 0, r_{\sigma} \rrbracket
$$

where

$$
r_{\sigma}=\frac{1-\sigma^{2}}{\sqrt{1+\sigma^{2}}}
$$

Theorem 27. If $0<\sigma<1$ then

$$
\{\lambda:|\lambda| \leq 1\} \backslash H_{\sigma} \subseteq S_{\sigma}
$$

Proof. Note first that if $c^{ \pm}$and $\rho_{n}^{ \pm}(\theta, \sigma)$ are defined as in Lemma 24, then

$$
\rho_{n}^{ \pm}(\theta, \sigma) \geq \rho_{\sigma, n}=\left(\frac{1-\sigma^{2^{n+1}}}{1+\sigma^{2^{n}}}\right)^{1 / 2^{n}},
$$

for all $\theta \in \mathbf{R}$, so that $I_{c^{ \pm}} \supset\left\{\lambda:|\lambda|<\rho_{\sigma, n}\right\}$. Thus, defining $c^{*} \in \Omega_{\sigma}$ as in Lemma 25, with $c=c^{+}$or $c^{-}$and $\tau= \pm \sigma$, we see from Lemma 25 that

$$
\operatorname{Spec}\left(A_{c^{*}}\right) \supset \overline{I_{c}} \backslash E_{\tau} \supset\left\{\lambda:|\lambda| \leq \rho_{\sigma, n}\right\} \backslash E_{\tau}
$$

Applying Proposition 1, it follows that, for all $n \in \mathbb{N}$,

$$
S_{\sigma} \supset\left\{\lambda:|\lambda| \leq \rho_{\sigma, n}\right\} \backslash H_{\tau}
$$

The theorem follows since $\sup _{n} \rho_{\sigma, n}=1$ and $S_{\sigma}$ is closed.

Lemma 26 and Theorem 27 together establish that there is a hole in $S_{\sigma}$ which is at least as big as $\{\lambda:|\lambda|<1-\sigma\}$ and which is no larger than $H_{\sigma}$. The numerical computations we have been able to carry out are consistent with a hypothesis that the hole is precisely the set $H_{\sigma}$, i.e. they are consistent with a hypothesis that $\operatorname{Spec}\left(A_{c}\right) \cap$ $H_{\sigma}=\emptyset$ for every $c \in \Omega_{\sigma}$, and hence for every $c \in E_{\sigma}$.

It should be pointed out, however, that these numerical computations are only for instances where $c$ is periodic, for which we have a characterisation of the spectrum in Lemma 20. Thus, strictly speaking, our calculations are evidence of the possibly 
weaker result that $\pi_{\infty, \sigma} \cap H_{\sigma}=\emptyset$; they become evidence that $\operatorname{Spec}\left(A_{c}\right) \cap H_{\sigma}=\varnothing$ with a hypothesis that $\pi_{\infty, \sigma}$ is dense in the part of $S_{\sigma}$ that is contained in the unit disc. This latter statement may or may not be true for $\sigma \in(0,1)$, but we have shown in Theorem 15 that it is true for $\sigma=1$.

As an example of the numerical computations we have carried out, the right hand side of Figure 1 shows the union of $\operatorname{Spec}\left(A_{c}\right)$ over all periodic $c \in \Omega_{\sigma}$ for which the period $N \leq 12$. It is clear from this figure that $\pi_{12, \sigma} \cap H_{\sigma}=\emptyset$ for $\sigma=0.5$. We note that, rather than using the characterisation in Lemma 20, we use for these computations the standard Bloch-decomposition formula (e.g. [11]) that

$$
\operatorname{Spec}\left(A_{c}\right)=\bigcup_{|\alpha|=1} \operatorname{Spec}\left(A_{c, \alpha}^{(N, \text { per })}\right),
$$

where $A_{c, \alpha}^{(N, \text { per) }}$ is the $N \times N$ matrix defined in (51) below.
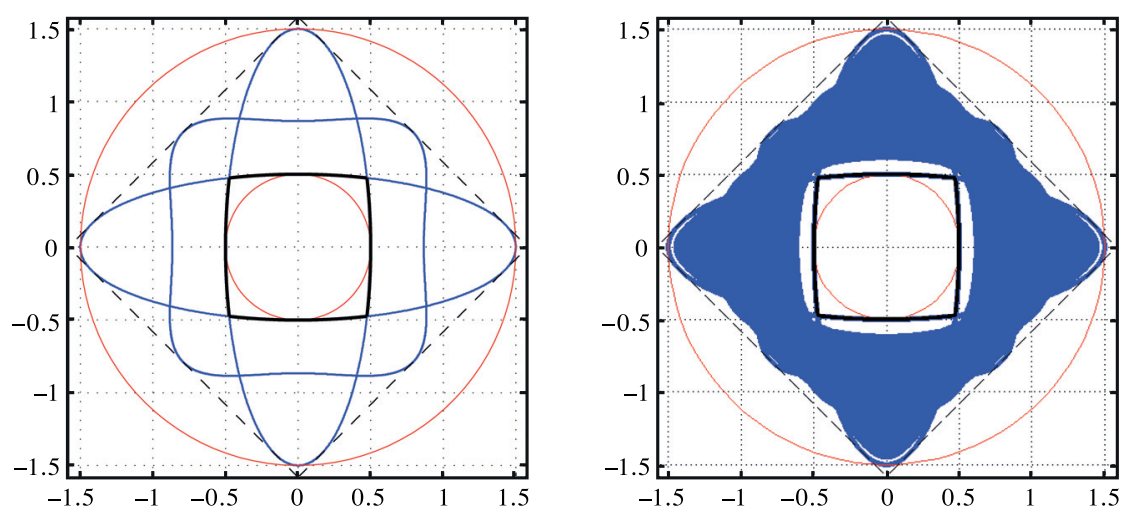

Figure 1. Plots of $\operatorname{Spec}\left(A_{c}\right)$ for the case when $c$ is periodic and $\sigma=0.5$. The two plots show the sets $\pi_{N, \sigma} \subset S_{\sigma}$, the union of the spectra for all sequences $c$ of period $\leq N$, for $N=2$ (left) and $N=12$ (right). The two ellipses visible in the left-hand plot, the boundaries of $E_{\sigma}$ and $E_{-\sigma}$ defined in (32) and (33), are the components of $\pi_{1, \sigma}$; the other closed curve is $\operatorname{Spec}\left(A_{c}\right)$ for $c=\sigma c^{(1,-)}$, i.e. $c_{n}=(-1)^{n} \sigma$, given explicitly in Lemma 24. Also shown in each plot are the boundaries of the inclusion sets from Lemma 26, namely the circles of radius $1 \pm \sigma$, and, in dashed lines, the boundary of the set (35). The boundary of $H_{\sigma}$, which we conjecture is a hole in the spectrum $S_{\sigma}$, is highlighted in a thicker line.

It is not feasible to calculate $\pi_{N, \sigma}$, the union of all $2^{N-1}$ periodic spectra of period $N$, for very much larger values of $N$. In Figure 2 we sample $\pi_{100, \sigma}$, for $\sigma=0.5$, plotting the union of the spectra of $10^{5}$ randomly chosen $N \times N$ matrices $A_{c, \alpha}^{(N, \text { per })}$. By randomly chosen we mean here that, in each realisation, $N \in\{1, \ldots, 100\}$ is randomly chosen, with higher probabilities for the smaller matrix sizes, and then the vector $c=\left(c_{1}, \ldots, c_{N}\right)$ is randomly chosen, with each $c_{n}= \pm \sigma$ independent and identically distributed with $\operatorname{Pr}\left(c_{n}=\sigma\right)=0.5$, and finally the phase factor $\alpha$ is 
randomly chosen from a uniform measure on the unit circle. We see in the figure that clearly, as they have to, the spectra are constrained to lie in the inclusion sets shown in Lemma 26. We also note that all the spectra lie outside $H_{\sigma}$.

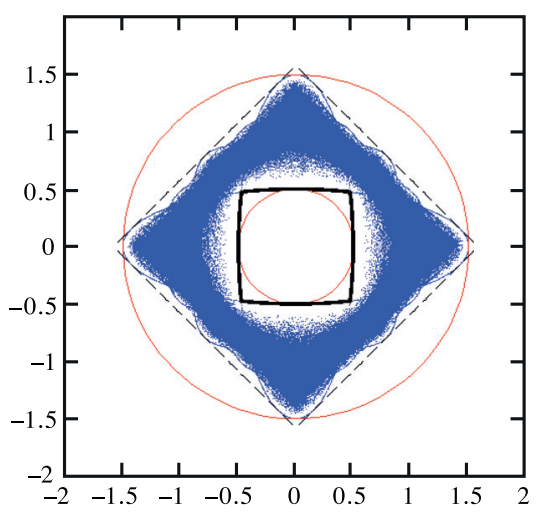

Figure 2. Plots of $10^{5}$ instances of $\operatorname{Spec}\left(A_{c, \alpha}^{(N, \text { per })}\right)$ for $\sigma=0.5$, computed as described in the text, with $N$ randomly chosen in the range $1 \leq N \leq 100$.

\section{Second proof of the main theorem}

In Theorem 34 of this section we provide a second proof that

$$
\{\lambda:|\lambda| \leq 1\} \backslash H_{\sigma} \subseteq S_{\sigma}
$$

for all $\sigma \in(0,1)$. This proof has a lot in common with the previous one, but it reveals more about the asymptotic behaviour of the solutions of the second order recurrence relation for certain choices of $c \in \Omega_{\sigma}$. A key role in this section is played by the sequence $c_{e} \in \Omega_{\sigma}$ defined in Section 4, which sequence was central to the proof of Theorem 7 that appears in [5] (see Theorem 12 above for more details).

We start with some calculations that do not depend on $\sigma$. Throughout this section $\tilde{c}_{n} \in\{ \pm 1\}$ is defined for all $n \geq 1$ by the rules $\tilde{c}_{1}=1, \tilde{c}_{2 n}=\tilde{c}_{2 n-1} \tilde{c}_{n}$, and $\tilde{c}_{2 n}+\tilde{c}_{2 n+1}=0$. (In other words, $\tilde{c}_{n}=c_{e, n}$, for $n \geq 1$.) The first few values are shown in Table 1 . We will obtain a bound on a transfer matrix $T_{m, \lambda}$ associated with this sequence and use this bound to prove Theorem 34 .

Let $u: \mathbb{Z}_{+} \rightarrow \mathbb{C}$ be the solution of $u_{n+1}=\lambda u_{n}-\tilde{c}_{n} u_{n-1}$ such that $u_{0}=0$ and $u_{1}=1$, so that $u$ is the restriction to $\mathbb{Z}_{+}$of the bi-infinite sequence $u_{e}$ already studied in [5] and discussed in Section 4. We have observed already in Proposition 11 that $u_{n}$ is a polynomial of degree $n-1$ in $\lambda$ with integer coefficients for all $n \geq 2$. Similarly, if $v: \mathbb{Z}_{+} \rightarrow \mathbb{C}$ is the solution of $v_{n+1}=\lambda v_{n}-\tilde{c}_{n} v_{n-1}$ such that $v_{0}=1$ and $v_{1}=0$, it is easy to see that $v_{n}$ is a polynomial of degree $n-2$ with integer coefficients for all $n \geq 2$. 
Table 1. Values of $\tilde{c}_{n}, u_{n}, v_{n}$ for $1 \leq n \leq 9$.

\begin{tabular}{rrrr}
$n$ & $\tilde{c}_{n}$ & $u_{n}$ & $v_{n}$ \\
\hline 1 & 1 & 1 & 0 \\
2 & 1 & $\lambda$ & -1 \\
3 & -1 & $\lambda^{2}-1$ & $-\lambda$ \\
4 & -1 & $\lambda^{3}$ & $-\lambda^{2}-1$ \\
5 & 1 & $\lambda^{4}+\lambda^{2}-1$ & $-\lambda^{3}-2 \lambda$ \\
6 & -1 & $\lambda^{5}-\lambda$ & $-\lambda^{4}-\lambda^{2}+1$ \\
7 & 1 & $\lambda^{6}+\lambda^{4}-1$ & $-\lambda^{5}-2 \lambda^{3}-\lambda$ \\
8 & -1 & $\lambda^{7}$ & $-\lambda^{6}-\lambda^{4}-1$ \\
9 & 1 & $\lambda^{8}+\lambda^{6}+\lambda^{4}-1$ & $-\lambda^{7}-2 \lambda^{5}-2 \lambda^{3}-2 \lambda$
\end{tabular}

One may check the computations of $u_{m}$ and $v_{m}$ in Table 1 by confirming the determinantal identity $u_{m} v_{m+1}-v_{m} u_{m+1}= \pm 1$ for all $m \geq 1$, the left hand side being a polynomial in $\lambda$. Here we are referring to a determinantal identity for the transfer matrix $T_{m, \lambda}$, defined by

$$
T_{m, \lambda}=\left(\begin{array}{cc}
v_{m} & u_{m} \\
v_{m+1} & u_{m+1}
\end{array}\right),
$$

which transfers the data of any solution of $x_{n+1}=\lambda x_{n}-\tilde{c}_{n} x_{n-1}$ from $\{0,1\}$ to $\{m, m+1\}$ in the sense that

$$
T_{m, \lambda}\left(\begin{array}{c}
x_{0} \\
x_{1}
\end{array}\right)=\left(\begin{array}{c}
x_{m} \\
x_{m+1}
\end{array}\right)
$$

It is easy to verify (see Lemma 20 above) that

$$
T_{m, \lambda}=X_{m} X_{m-1} \ldots X_{1}
$$

where

$$
X_{r}=\left(\begin{array}{cc}
0 & 1 \\
-\tilde{c}_{r} & \lambda
\end{array}\right)
$$

has determinant $\tilde{c}_{r} \in\{ \pm 1\}$ for every $r \geq 1$.

The proof of Theorem 34 we will give below was motivated by numerical evidence that

$$
\operatorname{tr}\left(T_{2^{r}, \lambda}\right)=\lambda^{2^{r}}-2
$$

holds for all $r \geq 1$ and all $\lambda \in \mathbb{C}$; see Table 2 . We prove this crucial identity in Lemma 31. 
Table 2. Values of $\operatorname{tr}\left(T_{n, \lambda}\right)$ for $1 \leq n \leq 8$.

\begin{tabular}{rr}
$n$ & $\operatorname{tr}\left(T_{n, \lambda}\right)=u_{n+1}+v_{n}$ \\
\hline 1 & $\lambda$ \\
2 & $\lambda^{2}-2$ \\
3 & $\lambda^{3}-\lambda$ \\
4 & $\lambda^{4}-2$ \\
5 & $\lambda^{3}-3 \lambda$ \\
6 & $\lambda^{6}-\lambda^{2}$ \\
7 & $\lambda^{5}-\lambda^{3}-\lambda$ \\
8 & $\lambda^{8}-2$
\end{tabular}

Lemma 28. Let $T$ be a $2 \times 2$ matrix with determinant $\delta$ and trace $\tau$. If $\tau^{2} \neq 4 \delta$ and $\gamma$ is the absolute value of the larger root of $z^{2}-\tau z+\delta=0$ then there exists $a$ constant $b$ such that

$$
\left\|T^{r}\right\| \leq b \gamma^{r}
$$

for all $r \geq 1$. If $\tau^{2}=4 \delta$ then for every $\varepsilon>0$ there exists a constant $b_{\varepsilon}$ such that

$$
\left\|T^{r}\right\| \leq b_{\varepsilon}(\gamma+\varepsilon)^{r}
$$

for all $r \geq 1$.

Proof. The eigenvalues $z_{ \pm}$of $T$ are the roots $z$ of $z^{2}-\tau z+\delta=0$. The condition $\tau^{2} \neq 4 \delta$ implies that the eigenvalues are distinct, so $T$ is diagonalizable - there exists an invertible matrix $B$ such that

$$
T=B\left(\begin{array}{cc}
z_{+} & 0 \\
0 & z_{-}
\end{array}\right) B^{-1}
$$

Therefore

$$
\left\|T^{r}\right\|=\left\|B\left(\begin{array}{cc}
z_{+}^{r} & 0 \\
0 & z_{-}^{r}
\end{array}\right) B^{-1}\right\| \leq\|B\|\left\|B^{-1}\right\| \gamma^{r} .
$$

The slightly worse bound (44) is obtained when $\tau^{2}=4 \delta$ because one has to use the Jordan canonical form for $T$.

Lemma 29. The identity $\operatorname{det}\left(T_{2^{n}, \lambda}\right)=1$ holds for all $n \geq 1$ and all $\lambda \in \mathbb{C}$.

Proof. If $m \in \mathbb{N}$ then (42) and (43) imply

$$
\operatorname{det}\left(T_{2 m, \lambda}\right)=\prod_{r=1}^{m} \operatorname{det}\left(X_{2 r} X_{2 r-1}\right)=\prod_{r=1}^{m}\left(\tilde{c}_{2 r} \tilde{c}_{2 r-1}\right)=\prod_{r=1}^{m} \tilde{c}_{r}=\operatorname{det}\left(T_{m, \lambda}\right) .
$$


It follows by induction that

$$
\operatorname{det}\left(T_{2^{n}, \lambda}\right)=\operatorname{det}\left(T_{1, \lambda}\right)=\tilde{c}_{1}=1
$$

The following lemma depends on Proposition 11 above, abstracted from [5], which notes properties of the integer coefficients $p_{i, j}$ of the polynomials

$$
u_{i}=\sum_{j=1}^{i} p_{i, j} \lambda^{j-1}
$$

Lemma 30. The polynomial $u_{m}$ is even for odd $m$ and odd for even $m$. Its leading term is $\lambda^{m-1}$. If $m=2^{n}$ and $n \geq 2$ then

$$
\begin{aligned}
u_{m} & =\lambda^{m-1}, \\
u_{m+1} & =-1+\lambda^{m / 2} \sum_{r=0}^{m / 4} \alpha_{r} \lambda^{2 r},
\end{aligned}
$$

where $\alpha_{r} \in\{0,1,-1\}$ for all $r$.

Proof. The statements in the first two sentences may be proved by induction, using the definition of $u_{m}$. We prove (45) and (46) for $m=2^{n}$ by induction in $n$, noting that both hold for $n \leq 3$; see Table 1. As in Proposition 11, let $Y$ denote the set of $(i, j) \in \mathbb{N}^{2}$ such that $p_{i, j} \neq 0$.

To prove (45) suppose that $\left(2^{n+1}, j\right) \in Y$. Proposition 11 implies that $j$ is even and that $\left(2^{n}, j / 2\right) \in Y$. The inductive hypothesis now implies that $j / 2=2^{n}$, so $j=2^{n+1}$.

To prove (46) suppose that $\left(2^{n+1}+1, j\right) \in Y$. Proposition 11 implies that $j$ is odd and either $\left(2^{n},(j+1) / 2\right) \in Y$ or $\left(2^{n}+1,(j+1) / 2\right) \in Y$. In the first case we have already proved that $(j+1) / 2=2^{n}$, so $j=2^{n+1}-1$. In the second case the inductive hypothesis implies that $(j+1) / 2 \geq 2^{n-1}+1$, so $j \geq 2^{n}+1$ or $(j+1) / 2=1$, so $j=1$.

We finally have to evaluate the constant coefficient $\gamma_{m}$ of $u_{m}$ when $m=2^{n}+1$. This may be done by considering the defining recurrence relation in the case $\lambda=0$, namely $\gamma_{r+1}=-\tilde{c}_{r} \gamma_{r-1}$ subject to $\gamma_{0}=0$ and $\gamma_{1}=1$. This implies that

$$
\gamma_{2 m+1}=\prod_{r=1}^{m} \tilde{c}_{2 r}
$$

for all $m \in \mathbb{N}$. Therefore $\gamma_{5}=\gamma_{3}=-1$ and

$$
\gamma_{8 m+1}=\prod_{r=1}^{m}\left(\tilde{c}_{8 r} \tilde{c}_{8 r-2} \tilde{c}_{8 r-4} \tilde{c}_{8 r-6}\right)
$$




$$
\begin{aligned}
& =\prod_{r=1}^{m}\left(\tilde{c}_{4 r} \tilde{c}_{8 r-1} \tilde{c}_{8 r-2} \tilde{c}_{4 r-2} \tilde{c}_{8 r-5} \tilde{c}_{8 r-6}\right) \\
& =\prod_{r=1}^{m}\left(\tilde{c}_{4 r} \tilde{c}_{4 r-2}\right) \\
& =\gamma_{4 m+1}
\end{aligned}
$$

for all $m \in \mathbb{N}$. A simple induction now implies that $\gamma_{m}=-1$ for $m=2^{n}+1$ and all $n \geq 1$.

Lemma 31. If $m=2^{n}$ and $n \geq 2$ then

$$
\tau=\operatorname{tr}\left(T_{m, \lambda}\right)=v_{m}+u_{m+1}=\lambda^{m}-2
$$

for all $\lambda \in \mathbb{C}$.

Proof. The proof uses the identity $v_{m} u_{m+1}-u_{m} v_{m+1}=1$ of Lemma 29 together with the two identities proved in Lemma 30. These are identities within the commutative ring $\mathbb{Z}(\lambda)$ of all polynomials with integer coefficients in the indeterminate quantity $\lambda$, but they imply similar identities in the commutative ring $\mathbb{Z}(\hat{\lambda})$ of all polynomials with integer coefficients in an indeterminate quantity $\hat{\lambda}$ that satisfies the identity $\hat{\lambda}^{m-1}=0$. (Equivalently one may start by disregarding all terms in the identities that involve $\lambda^{r}$ with $r \geq m-1$.) The identities then simplify to

$$
\begin{aligned}
\hat{u}_{m+1} & =-1+p, \\
\hat{v}_{m} \hat{u}_{m+1} & =1,
\end{aligned}
$$

where

$$
p(\hat{\lambda})=\hat{\lambda}^{m / 2} \sum_{r=0}^{m / 4} \alpha_{r} \hat{\lambda}^{2 r}
$$

satisfies $p^{2}=0$ in $\mathbb{Z}(\hat{\lambda})$. The second equation can be solved for $\hat{v}_{m}$, yielding

$$
\hat{v}_{m}=-1-p
$$

and hence $\hat{\tau}=-2$. Returning to the original variable $\lambda$ one deduces that

$$
\tau=-2+\sum_{r \geq m-1} \beta_{r} \lambda^{r} .
$$

But (cf. Lemma 30) it is easily shown that $v_{n}$ is an even polynomial of degree $n-2$ for odd $n$. Thus, and by Lemma 30, $\tau=v_{m}+u_{m+1}$ is an even polynomial of degree $m$ with leading coefficient 1 , so $\tau=\lambda^{m}-2$. 
Lemma 32. Following the assumptions and notation of Lemma 28, suppose that $\delta=1$ and that there exist $m \in \mathbb{Z}_{+}$and $\mu \in \mathbb{C}$ such that $\tau=\mu^{m}-2$. Then there exists a constant $b$ such that

$$
\left\|T^{r}\right\| \leq b 4^{r} \max \left(|\mu|^{r m}, 1\right)
$$

for all $r \geq 1$.

Proof. Case 1. If $|\mu| \leq 1$ it suffices to obtain bounds on the solutions $z_{ \pm}$of $z^{2}-$ $\tau z+1=0$ when $|\tau| \leq 3$. The solutions satisfy

$$
\left|z_{ \pm}\right|=\left|\frac{\tau}{2} \pm \sqrt{\frac{\tau^{2}}{4}-1}\right| \leq \frac{3}{2}+\frac{\sqrt{13}}{2}<4 .
$$

Lemma 28 now implies that $\left\|T^{r}\right\| \leq b 4^{r}$ for all $r \geq 1$.

Case 2. If $|\mu|>1$ it suffices to obtain bounds on the solutions $z_{ \pm}$of $z^{2}-\tau z+1=$ 0 when $|\tau| \leq 3|\mu|^{m}$. The solutions satisfy

$$
\left|z_{ \pm}\right|=\left|\frac{\tau}{2} \pm \sqrt{\frac{\tau^{2}}{4}-1}\right|<4|\mu|^{m} .
$$

Lemma 28 now implies that $\left\|T^{r}\right\| \leq b 4^{r}|\mu|^{r m}$ for all $r \geq 1$.

Lemma 33. Let $X_{n}, n \in \mathbb{Z}$, be a periodic sequence of $2 \times 2$ matrices with period $m$ and let $T_{r}=X_{r} X_{r-1} \ldots X_{1}$ for all $r \geq 1$. If there exist constants $b_{0}, \gamma$ such that $\left\|\left(T_{m}\right)^{s}\right\| \leq b_{0} \gamma^{s}$ for all $s \geq 0$, then there exists a constant $b_{2}$ such that $\left\|T_{r}\right\| \leq$ $b_{2} \gamma^{r / m}$ for all $r \geq 1$.

Proof. Every $r \in \mathbb{Z}_{+}$may be written in the form $r=s m+v$ where $s \geq 0$ and $0 \leq v<m$. Using the identity $T_{s m}=\left(T_{m}\right)^{s}$, one obtains

$$
\begin{aligned}
\left\|T_{r}\right\| & =\left\|X_{r} X_{r-1} \ldots X_{s m+1} T_{s m}\right\| \\
& =\left\|X_{v} X_{v-1} \ldots X_{1}\left(T_{m}\right)^{s}\right\| \\
& \leq\left\|X_{v} X_{v-1} \ldots X_{1}\right\| b_{0} \gamma^{s m} \\
& \leq b_{0} b_{1} \gamma^{s} \\
& \leq b_{2} \gamma^{r / m}
\end{aligned}
$$

where $b_{2}=b_{0} b_{1}$ and

$$
b_{1}=\max _{0 \leq v \leq m-1}\left\{\left\|X_{v} X_{v-1} \ldots X_{1}\right\|\right\} .
$$


Theorem 34. One has

$$
\{\lambda:|\lambda| \leq 1\} \backslash H_{\sigma} \subseteq S_{\sigma}
$$

for all $\sigma \in(0,1)$.

Proof. Given $\sigma \in(0,1)$ we put $m=2^{d}$ where $d \in \mathbb{N}$ is large enough to yield

$$
\sigma^{1 / 2}<h=4^{-1 / m} \text {. }
$$

We use the identities

$$
\delta=\operatorname{det}\left(T_{m, \mu}\right)=1
$$

and

$$
\tau=\operatorname{tr}\left(T_{m, \mu}\right)=\mu^{m}-2
$$

proved in Lemmata 29 and 31 and valid for all $\mu \in \mathbb{C}$. Let $c \in \Omega_{\sigma}$ be the periodic sequence with period $m$ such that $c_{n}=\sigma \tilde{c}_{n}$ for all $1 \leq n \leq m$. The main task is to prove that if $|\lambda|<h$ then all solutions $\xi: \mathbb{Z} \rightarrow \mathbb{C}$ of

$$
\xi_{n+1}=\lambda \xi_{n}-c_{n} \xi_{n-1}
$$

decay exponentially as $n \rightarrow+\infty$. This will imply, by Lemma 20, and using the notations of that lemma, that

$$
I_{c} \supset\{\lambda:|\lambda|<h\} .
$$

Arguing as in the proof of Theorem 27, it will then follow from Lemma 25 and Proposition 1 that

$$
S_{\sigma} \supset\{\lambda:|\lambda| \leq h\} \backslash H_{\sigma},
$$

this holding for any $h=4^{-1 / m}$ such that (49) holds and $m=2^{d}$, so that

$$
S_{\sigma} \supset\{\lambda:|\lambda|<1\} \backslash H_{\sigma} .
$$

Since $S_{\sigma}$ is closed, (48) will follow.

Thus it remains only to show that all solutions of (50) decay exponentially at $+\infty$. To see that this holds, define $x_{n}=\sigma^{-n / 2} \xi_{n}$ and $\mu=\sigma^{-1 / 2} \lambda$ so that (50) may be rewritten in the form

$$
x_{n+1}=\mu x_{n}-\tilde{c}_{n} x_{n-1}
$$

for $1 \leq n \leq m$. Where $\theta=\max (1,|\mu|)$, Lemma 32 now yields

$$
\left\|\left(T_{m, \mu}\right)^{r}\right\| \leq b 4^{r} \theta^{r m}
$$

for all $r \in \mathbb{N}$. Lemma 33 with $\gamma=4 \theta^{m}$ implies

$$
\left\|T_{r, \mu}\right\| \leq b 4^{r / m} \theta^{r},
$$

and hence

$$
\left|x_{r}\right| \leq b_{3} 4^{r / m} \theta^{r}
$$

again for all $r \in \mathbb{N}$. Hence, where $\varphi=\max \left(\sigma^{1 / 2},|\lambda|\right)$,

$$
\left|\xi_{r}\right| \leq b_{3} 4^{r / m} \theta^{r} \sigma^{r / 2}=b_{3}\left(\varphi h^{-1}\right)^{r}
$$

for all $r \in \mathbb{N}$. Since $0<\varphi<h$, it follows that $\xi$ decays exponentially. 


\section{Semi-infinite and finite matrices}

All our results so far have focused on calculations of the spectrum of the bi-infinite matrix $A_{c}$. In this final section we say something about the spectrum of the semiinfinite matrix

$$
A_{c}^{+}=\left(\begin{array}{cccc}
0 & 1 & & \\
c_{1} & 0 & 1 & \\
& c_{2} & 0 & \ddots \\
& & \ddots & \ddots
\end{array}\right)
$$

in the case that $c=\left(c_{1}, c_{2}, \ldots\right) \in\{ \pm \sigma\}^{\mathbb{N}}$ is pseudo-ergodic (contains every finite sequence of $\pm \sigma$ 's as a consecutive sequence). We also say something (though have mainly unanswered questions) about the finite $N \times N$ matrices

$$
A_{c}^{(N)}=\left(\begin{array}{ccccc}
0 & 1 & & & \\
c_{1} & 0 & 1 & & \\
& c_{2} & 0 & \ddots & \\
& & \ddots & \ddots & 1 \\
& & & c_{N-1} & 0
\end{array}\right)
$$

and

$$
A_{c, \alpha}^{(N, \text { per })}=\left(\begin{array}{ccccc}
0 & 1 & & & \alpha c_{N} \\
c_{1} & 0 & 1 & & \\
& c_{2} & 0 & \ddots & \\
& & \ddots & \ddots & 1 \\
\alpha^{-1} & & & c_{N-1} & 0
\end{array}\right) .
$$

Here $A_{c}^{(N)}$ is tridiagonal, $A_{c}^{(N, \text { per })}$ is tridiagonal except for "periodising" entries in row 1 column $N$ and row $N$ column 1 (in these entries we assume that $|\alpha|=1$ ), and each $c_{j}= \pm \sigma$ : we have in mind particularly the random case where the $c_{j}$ 's are independent and identically distributed random variables taking the values $\pm \sigma$.

Our main result on the spectrum of $A_{c}$, proved in the previous sections, is that it contains the set $\{\lambda:|\lambda| \leq 1\} \backslash H_{\sigma}$. We suspect that $H_{\sigma}$ is a genuine hole in the spectrum for $0<\sigma<1$, i.e. that $H_{\sigma} \cap S_{\sigma}=\emptyset$. We have not shown this result but have shown in Lemma 26 the weaker result that $\{\lambda:|\lambda|<1-\sigma\} \cap S_{\sigma}=\emptyset$. Our first result in this section is that this hole is not present in the spectrum of the semi-infinite matrix. The proof depends on recent results on semi-infinite pseudoergodic operators due to Lindner and Roch [19], derived using characterisations of the indices of those Fredholm operators whose matrix representations are banded semi-infinite matrices in terms of so-called "plus indices" of limit operators, these characterisations derived using $K$-theory results for $C^{*}$-algebras in [20]. 
Theorem 35. Suppose $c \in\{ \pm \sigma\}^{\mathbb{N}}$ is pseudo-ergodic. If $\sigma=1$ then $\operatorname{Spec}\left(A_{c}^{+}\right)=S$. For all $\sigma \in(0,1],\{\lambda:|\lambda| \leq 1\} \subset \operatorname{Spec}\left(A_{c}^{+}\right)$.

Proof. In the case that $\sigma=1$ it is shown in [6] that $\operatorname{Spec}\left(A_{c}^{+}\right)=S$. Thus, for $\sigma=1$,

$$
\{\lambda:|\lambda| \leq 1\} \subset S=\operatorname{Spec}\left(A_{c}^{+}\right)
$$

follows from Theorem 7 (or [5], Theorem 2.3). For all $\sigma \in(0,1]$ it follows from [19], Theorem 2.1, that the essential spectrum of $A_{c}^{+}$, i.e. the set of $\lambda \in \mathbb{C}$ for which $A_{c}^{+}-\lambda I^{+}$is not Fredholm (here $I^{+}$is the identity operator on $\ell^{2}(\mathbb{N})$ ), is the set $S_{\sigma}$. Thus and by Theorem 27,

$$
\left(\{\lambda:|\lambda| \leq 1\} \backslash H_{\sigma}\right) \subset S_{\sigma} \subset \operatorname{Spec}\left(A_{c}^{+}\right) .
$$

It remains to show that $H_{\sigma} \subset \operatorname{Spec}\left(A_{c}^{+}\right)$. But, applying Theorem 2.4 in [19] (note that the set $E_{-}(U, W)$ in the notation of Theorem 2.4 in [19] is precisely the set $H_{\sigma}$ for this operator), it follows that, for $\lambda \in H_{\sigma}$, either $A_{c}^{+}-\lambda I^{+}$is not Fredholm or $A_{c}^{+}-\lambda I^{+}$is Fredholm with index 1: in either of these cases $\lambda \in \operatorname{Spec}\left(A_{c}^{+}\right)$.

Our other result in this section is to say something about the spectra (sets of eigenvalues) of the finite matrices $A_{c}^{(N)}$ and $A_{c, \alpha}^{(N, \text { per })}$. The notations $\pi_{N, \sigma}$ and $\pi_{\infty, \sigma}$ are as defined in and above equation (1) (and $\pi_{N}$ and $\pi_{\infty}$ are our abbreviations for $\sigma=1)$.

Theorem 36. If $0<\sigma \leq 1,|\alpha|=1$ and $c \in\{ \pm \sigma\}^{N}$, then

$$
\operatorname{Spec}\left(A_{c, \alpha}^{(N, \text { per })}\right) \subset \pi_{N, \sigma} \subset \pi_{\infty, \sigma} \subset S_{\sigma}
$$

while

$$
\operatorname{Spec}\left(A_{c}^{(N)}\right) \subset \sqrt{\sigma} \pi_{2 N+2} \subset \sqrt{\sigma} \pi_{\infty} \subset \sqrt{\sigma} S .
$$

If $\lambda=x+\mathrm{i} y$ is an eigenvalue of $A_{c, \alpha}^{(N, \text { per })}$ then $1-\sigma \leq|\lambda| \leq 1+\sigma$ and $|x|+|y| \leq$ $\sqrt{2\left(1+\sigma^{2}\right)}$, while if $\lambda$ is an eigenvalue of $A_{c}^{(N)}$ then $|x|+|y| \leq 2 \sqrt{\sigma}$.

Proof. The first of these statements is clear from the definition of $\pi_{N, \sigma}$, eq. (40), and Proposition 1 which gives that $\pi_{\infty, \sigma} \subset S_{\sigma}$. The second of these statements is shown for $\sigma=1$ in [6], Theorem 4.1. The second statement follows for $0<$ $\sigma<1$ by the observation that, where $d \in\{ \pm 1\}^{N}, c=\sigma d \in\{ \pm \sigma\}^{N}$, and $D_{N}$ is the diagonal matrix with leading diagonal $\left(1, \sigma^{1 / 2}, \sigma, \ldots, \sigma^{(N-1) / 2}\right)$, it holds that $D_{N}^{-1} A_{c}^{(N)} D_{N}=\sqrt{\sigma} A_{d}^{(N)}$. The last sentence then follows from Lemma 26.

Note that in the last sentence of the above theorem the condition $|x|+|y| \leq 2 \sqrt{\sigma}$ implies both that $|\lambda| \leq 1+\sigma$ and that $|x|+|y| \leq \sqrt{2\left(1+\sigma^{2}\right)}$.

In Figure 3 we plot the spectra of $A_{c}^{(N)}$ and $A_{c, \alpha}^{(N, \text { per })}$ for $N=2000$ and $\alpha=1$ for a typical realisation with the entries $c \in\{ \pm \sigma\}^{N}$ randomly chosen with the $c_{j}$ 
independently and identically distributed with $\operatorname{Pr}\left(c_{j}=\sigma\right)=0.5$ and $\sigma=0.9025$ so that $\sqrt{\sigma}=0.95$ (the several other realisations we have computed are very close in appearance to these plots). Theorem 36 tells us that $\operatorname{Spec}\left(A_{c}^{(N)}\right) \subset 0.95 \pi_{\infty} \subset 0.95 S$ and that $\operatorname{Spec}\left(A_{c, \alpha}^{(N, \text { per })}\right) \subset S_{0.9025}$, and that if $\lambda=x+$ i $y$ is an eigenvalue of $A_{c}^{(N)}$ then $|x|+|y| \leq 1.9$, while if $\lambda$ is an eigenvalue of $A_{c, \alpha}^{(N, \text { per })}$ then $0.075 \leq|\lambda| \leq 1.9025$ and $|x|+|y| \leq \sqrt{2\left(1+\sigma^{2}\right)} \approx 1.905$.
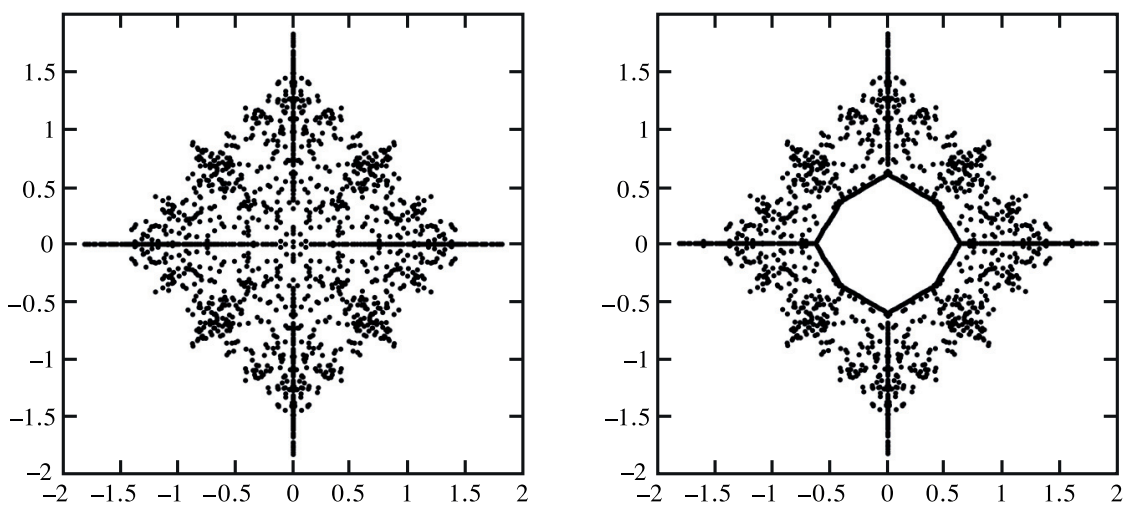

Figure 3. Plots of $\operatorname{Spec}\left(A_{c}^{(N)}\right)$ (left) and $\operatorname{Spec}\left(A_{c, \alpha}^{(N, \text { per })}\right)$ (right) for a case when $N=2000$, $\sigma=0.9025, \alpha=1$, and the entries of the vector $c=\left(c_{1}, \ldots, c_{N}\right)$ are independent and identically distributed with $\operatorname{Pr}\left(c_{j}= \pm \sigma\right)=0.5$ for each $j$ (the same vector $c$ is used in the two plots).

It is clear from Figure 3 that Theorem 36 is only the beginning of the story. We observe in the figure a hole in the spectrum of $A_{c, \alpha}^{(N, \text { per })}$, but it is a hole of radius approximately 0.6 , not 0.075 , with a large proportion of the eigenvalues positioned on the boundary of this hole, while outside the hole the spectra of $A_{c, \alpha}^{(N \text {,per })}$ and $A_{c}^{(N)}$ appear near identical. The same qualitative behaviour is visible in Figure 4, which is a similar plot except that $\sigma$ is reduced to 0.5 and we change the probability distribution, making it twice as likely that the entries of the vector $c$ are $-\sigma$ rather than $\sigma$. This change of probability distribution introduces an asymmetry, in particular an asymmetry in the hole in the spectrum (if we instead compute with $\operatorname{Pr}\left(c_{j}=\sigma\right)=1 / 2$ then typical realisations have spectra which are approximately invariant under the dihedral symmetry group $D_{2}$ of the square). Of course our methods, which are not probabilistic, have nothing to say about such asymmetries, indeed nothing, beyond Theorem 36 , to say about the almost sure spectra of $A_{c}^{(N)}$ or $A_{c, \alpha}^{(N, \text { per })}$ as $N \rightarrow \infty$. 

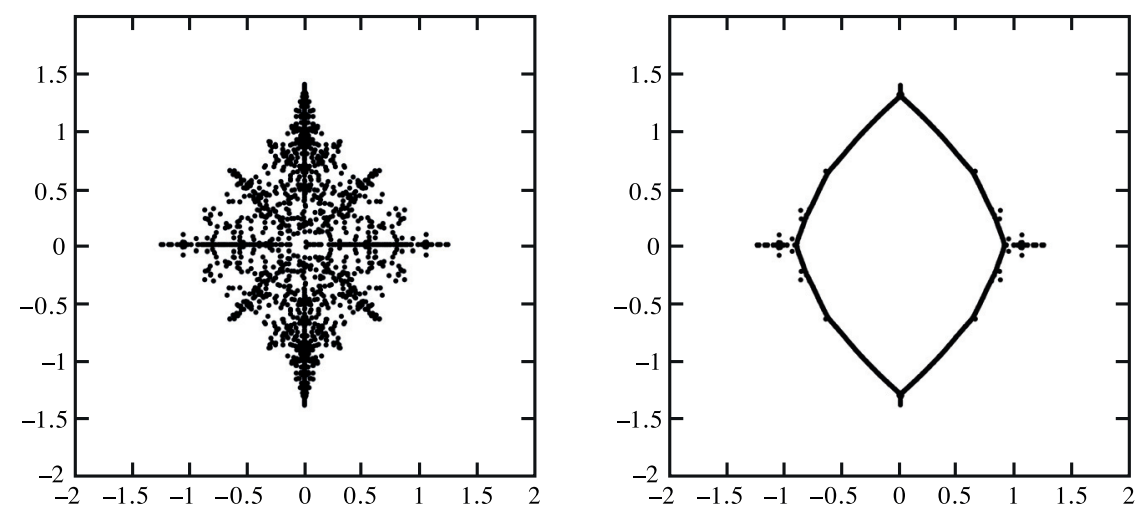

Figure 4. Plots of $\operatorname{Spec}\left(A_{c}^{(N)}\right)$ (left) and $\operatorname{Spec}\left(A_{c, \alpha}^{(N, \text { per })}\right)$ (right) for a case when $N=2000$, $\sigma=0.5$ and the entries of the vector $c=\left(c_{1}, \ldots, c_{N}\right)$ are independent and identically distributed with $\operatorname{Pr}\left(c_{j}=\sigma\right)=1 / 3$.

\section{References}

[1] A. Böttcher, Infinite matrices and projection methods. In A. Böttcher et al. (eds.), Lectures on operator theory and its applications. Fields Inst. Monogr. 3. Amer. Math. Soc., Providence (RI), 1996, 1-72. MR 1364444 Zbl 0842.65032

[2] A. Böttcher and Y. I. Karlovich, Carleson curves, Muckenhoupt weights, and Toeplitz operators. Birkhäuser, Basel, 1997. MR 1484165 Zbl 0889.47001

[3] A. Böttcher and B. Silbermann, Introduction to large truncated Toeplitz matrices. Springer Verlag, New York, 1999. MR 1724795 Zbl 0916.15012

[4] A. Böttcher and B. Silbermann, Analysis of Toeplitz operators. (second ed.). Prepared jointly with Alexei Karlovich. Springer Verlag, Berlin, 2006. MR 2223704 Zbl 1098.47002

[5] S. N. Chandler-Wilde, R. Chonchaiya, and M. Lindner, Eigenvalue problem meets Sierpinski triangle: computing the spectrum of a non-self-adjoint random operator. Oper. Matrices. 5 (2011), 633-648.

[6] S. N. Chandler-Wilde, R. Chonchaiya, and M. Lindner, On the spectra and pseudospectra of a class of non-self-adjoint random matrices and operators. Preprint 2011. arXiv:1107.0177v1

[7] S. N. Chandler-Wilde and M. Lindner, Limit operators, collective compactness, and the spectral theory of infinite matrices. Mem. Am. Math. Soc. 210 (2011), no. 986.

MR 2790742 Zbl 1219.47001

[8] G. M. Cicuta, M. Contedini, and L. Molinari, Non-Hermitian tridiagonal random matrices and returns to the origin of a random walk. J. Stat. Phys. 98 (2000), 685-699. MR 1749228 Zbl 0991.82015 
[9] E. B. Davies, Spectral properties of random non-self-adjoint matrices and operators. Proc. R. Soc. Lond., Ser. A, Math. Phys. Eng. Sci. 457 (2001), 191-206. MR 1843941 Zbl 1014.47002 rspa.royalsocietypublishing.org/content/457/2005/191

[10] E. B. Davies, Spectral theory of pseudo-ergodic operators. Commun. Math. Phys. 216 (2001), 687-704. MR 1815722 Zbl 1044.47031

[11] E. B. Davies, Linear operators and their spectra. Cambridge University Press, Cambridge, 2007. MR 2359869 Zbl 1138.47001

[12] E. B. Davies and B. Simon, Scattering theory for systems with different spatial asymptotics on the left and right. Commun. Math. Phys. 63 (1978), 277-301. MR 0513906 Zbl 0393.34015 projecteuclid.org/euclid.cmp/1103904569

[13] J. Feinberg and A. Zee, Non-Hermitean localization and de-localization. Phys. Rev. E 59 (1999), 6433-6443.

[14] J. Feinberg and A. Zee, Spectral curves of non-Hermitian Hamiltonians. Nucl. Phys. B552 (1999), 599-623. MR 1704620 Zbl 0944.82017

[15] I. Y. Goldsheid and B. A. Khoruzhenko, Distribution of eigenvalues in non-Hermitian Anderson model. Phys. Rev. Lett. 80 (1998), 2897-2901.

[16] I. Y. Goldsheid and B. A. Khoruzhenko, Thouless formula for random non-Hermitian Jacobi matrices. Isr. J. Math. 148 (2005), 331-346. MR 2191234 Zbl 1185.15034

[17] D. E. Holz, H. Orland, and A. Zee, On the remarkable spectrum of a non-Hermitian random matrix model. J. Phys. A, Math. Gen. 36 (2003), 3385-3400. MR 1986425 Zbl 1038.15016

[18] M. Lindner, Infinite matrices and their finite sections: An introduction to the limit operator method. Birkhäuser, Basel, 2006. MR 2253942 Zbl 1107.47001

[19] M. Lindner and S. Roch, Finite sections of random Jacobi operators. SIAM J. Numer. Anal. 50 (2012), 287-306.

[20] V. S. Rabinovich, S. Roch, and J. Roe, Fredholm indices of band-dominated operators. Integral Equations Oper. Theory 49 (2004), 221-238. MR 2060373 Zbl 1068.47016

[21] V. S. Rabinovich, S. Roch, and B. Silbermann, Limit operators and their applications in operator theory. Birkhäuser, Basel, 2004. MR 2075882 Zbl 1077.47002

Received October 3, 2011; revised December 23, 2011

S. N. Chandler-Wilde, Department of Mathematics and Statistics, University of Reading, Reading, RG6 6BB, U.K.

E-mail: s.n.chandler-wilde@ reading.ac.uk

E. B. Davies, Department of Mathematics, King's College London, Strand, London, WC2R 2LS, U.K.

E-mail: e.brian.davies@kcl.ac.uk 